Proceedings of RIKEN BNL Research Center Workshop Volume 93

\title{
PHENIX Spinfest School 2009 at BNL
}

\author{
July 01 - July 31,2009
}

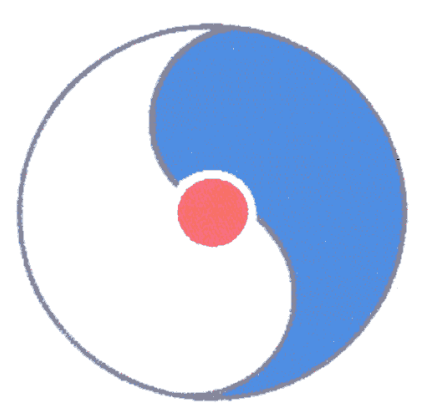

Organizers:

Ralf Christian Seidl, Yuji Goto, Kensuke Okada

RIKEN BNL Research Center

Building 510A, Brookhaven National Laboratory, Upton, NY 11973-5000, USA 


\section{DISCLAIMER}

This work was prepared as an account of work sponsored by an agency of the United States Government. Neither the United States Government nor any agency thereof, nor any of their employees, nor any of their contractors, subcontractors or their employees, makes any warranty, express or implied, or assumes any legal liability or responsibility for the accuracy, completeness, or any third party's use or the results of such use of any information, apparatus, product, or process disclosed, or represents that its use would not infringe privately owned rights. Reference herein to any specific commercial product, process, or service by trade name, trademark, manufacturer, or otherwise, does not necessarily constitute or imply its endorsement, recommendation, or favoring by the United States Government or any agency thereof or its contractors or subcontractors. The views and opinions of authors expressed herein do not necessarily state or reflect those of the United States Government or any agency thereof.

Notice: This manuscript has been authored by employees of Brookhaven Science Associates, LLC under Contract No. DE-AC02-98CH10886 with the U.S. Department of Energy. The publisher by accepting the manuscript for publication acknowledges that the United States Government retains a non-exclusive, paid-up, irrevocable, world-wide license to publish or reproduce the published form of this manuscript, or allow others to do so, for United States Government purposes. 


\section{Preface to the Series}

The RIKEN BNL Research Center (RBRC) was established in April 1997 at Brookhaven National Laboratory. It is funded by the "Rikagaku Kenkyusho" (RIKEN, The Institute of Physical and Chemical Research) of Japan. The Memorandum of Understanding between RIKEN and BNL, initiated in 1997, has been renewed in 2002 and again in 2007. The Center is dedicated to the study of strong interactions, including spin physics, lattice QCD, and RHIC physics through the nurturing of a new generation of young physicists.

The RBRC has both a theory and experimental component. The RBRC Theory Group and the RBRC Experimental Group consists of a total of 25-30 researchers. Positions include the following: full time RBRC Fellow, half-time RHIC Physics Fellow, and full-time, post-doctoral Research Associate. The RHIC Physics Fellows hold joint appointments with RBRC and other institutions and have tenure track positions at their respective universities or BNL. To date, RBRC has $\sim 50$ graduates of which 14 theorists and 6 experimenters have attained tenure positions at major institutions worldwide.

Beginning in 2001 a new RIKEN Spin Program (RSP) category was implemented at RBRC. These appointments are joint positions of RBRC and RIKEN and include the following positions in theory and experiment: RSP Researchers, RSP Research Associates, and Young Researchers, who are mentored by senior RBRC Scientists. A number of RIKEN Jr. Research Associates and Visiting Scientists also contribute to the physics program at the Center.

RBRC has an active workshop program on strong interaction physics with each workshop focused on a specific physics problem. In most cases all the talks are made available on the RBRC website. In addition, highlights to each speaker's presentation are collected to form proceedings which can therefore be made available within a short time after the workshop. To date there are ninety- two proceeding volumes available.

A 10 teraflops RBRC QCDOC computer funded by RIKEN, Japan, was unveiled at a dedication ceremony at BNL on May 26, 2005. This supercomputer was designed and built by individuals from Columbia University, IBM, BNL, RBRC, and the University of

Edinburgh, with the U.S. D.O.E. Office of Science providing infrastructure support at BNL. Physics results were reported at the RBRC QCDOC Symposium following the dedication. QCDSP, a 0.6 teraflops parallel processor, dedicated to lattice QCD, was begun at the Center on February 19, 1998, was completed on August 28, 1998, and was decommissioned in 2006. It was awarded the Gordon Bell Prize for price performance in 1998.

N. P. Samios, Director

June 2009

*Work performed under the auspices of U.S.D.O.E. Contract No. DE-AC02-98CH10886. 


\section{CONTENTS}

Preface to the Series

\section{PHENIX Spinfest School 2009 at BNL}

Introduction and Overview

Organizers: Ralf Seidl (BNL/RBRC); Yuji Goto (RIKEN/RBRC); Kensuke Okada i $(B N L / R B R C)$

eP scattering

Elke Aschenauer, BNL.............................................

PP scattering (and $\mathrm{e}+\mathrm{e}$ )

Matthias Grosse-Perdekamp, UIUC.................................. 8

Triggering in high energy experiments

John Lajoie, ISU.................................................. 16

Pythia and the Lund Fragmentation Model

Helen Caines, Yale................................................... 22

QCD Theory

Markus Diehl, DESY

List of Registered Participants

Agenda.

Group Photo.

38

Additional RIKEN-BNL Research Center Proceeding Volumes

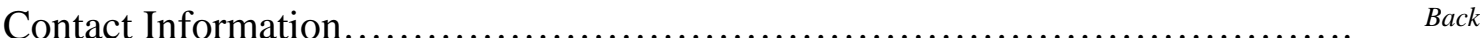




\section{INTRODUCTION}

Fifth Annual PHENIX Spinfest and School

Since 2005, the PHENIX Spin Physics Working Group has set aside several weeks each summer for the purposes of training and integrating recent members of the working group as well as coordinating and making rapid progress on support tasks and data analysis. One week is dedicated to more formal didactic lectures by outside speakers. The location has so far alternated between BNL and the RI KEN campus in Wako, Japan, with support provided by RBRC and LANL. Originally this year's Spinfest School was slated to take place in Japan, however, due to the H1N1 virus; this year's PHENIX Spinfest School 2009 will take place J uly 01 - 31, 2009 at BNL.

The mornings of July 7, 8, 9, and 13th lectures will take place in Bldg. 510 Small Seminar Room, Physics bldg. 510. The mornings of July, $27-31$, BIdg 510 Small Seminar room, the invited speaker will be Dr. Markus Diehl, DESY. All are welcome.

The Organizers

July 2009 

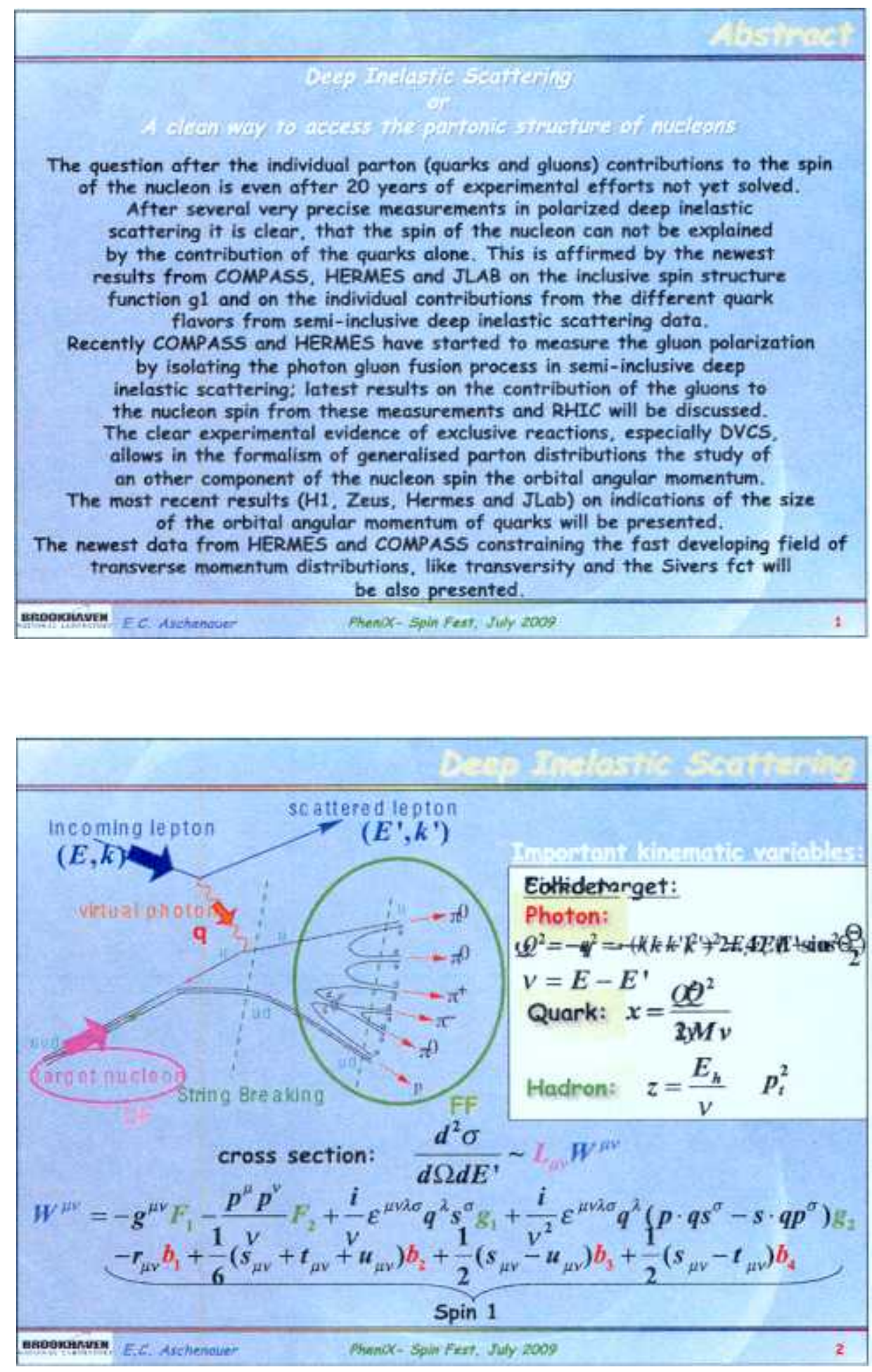

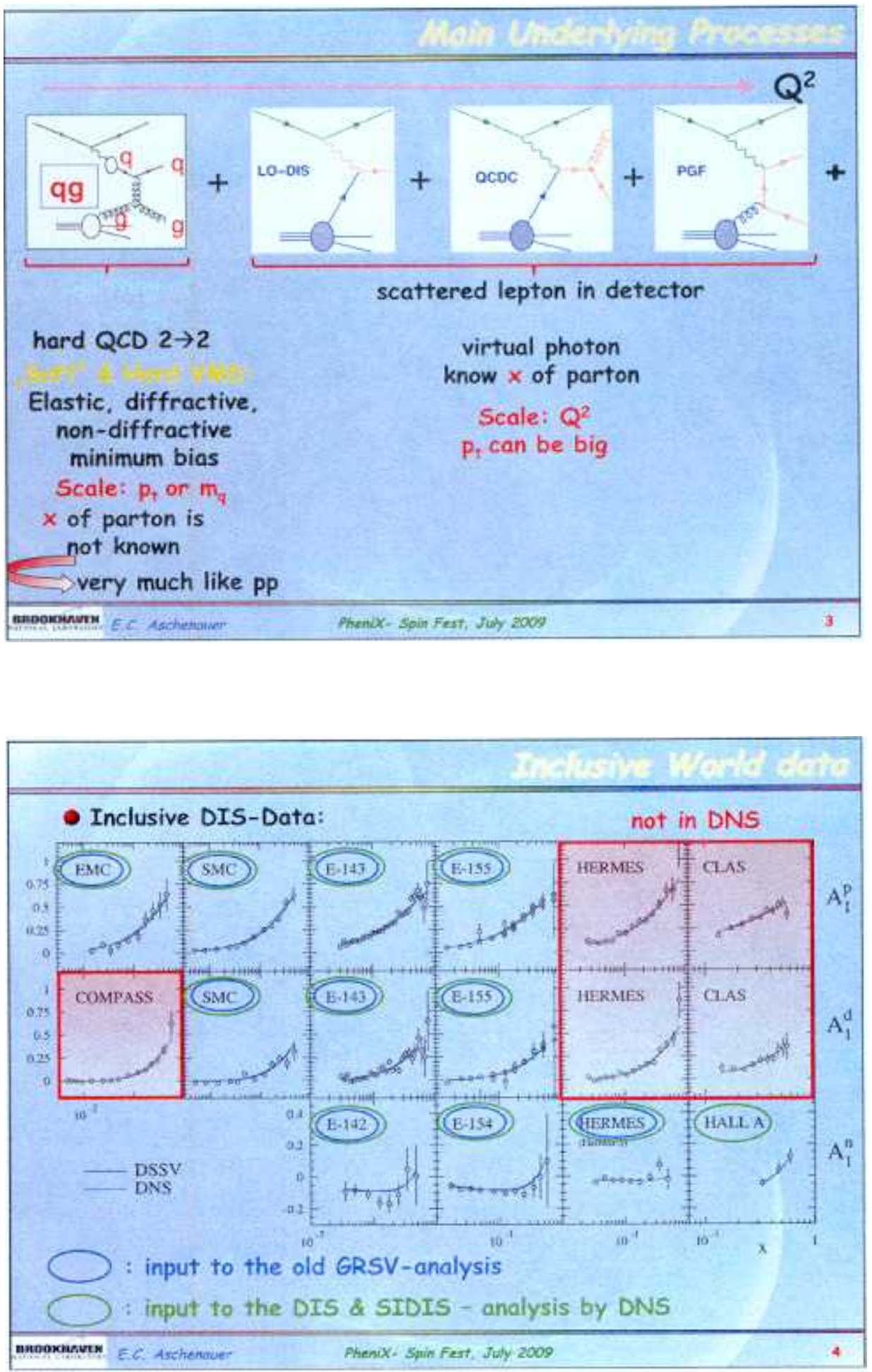

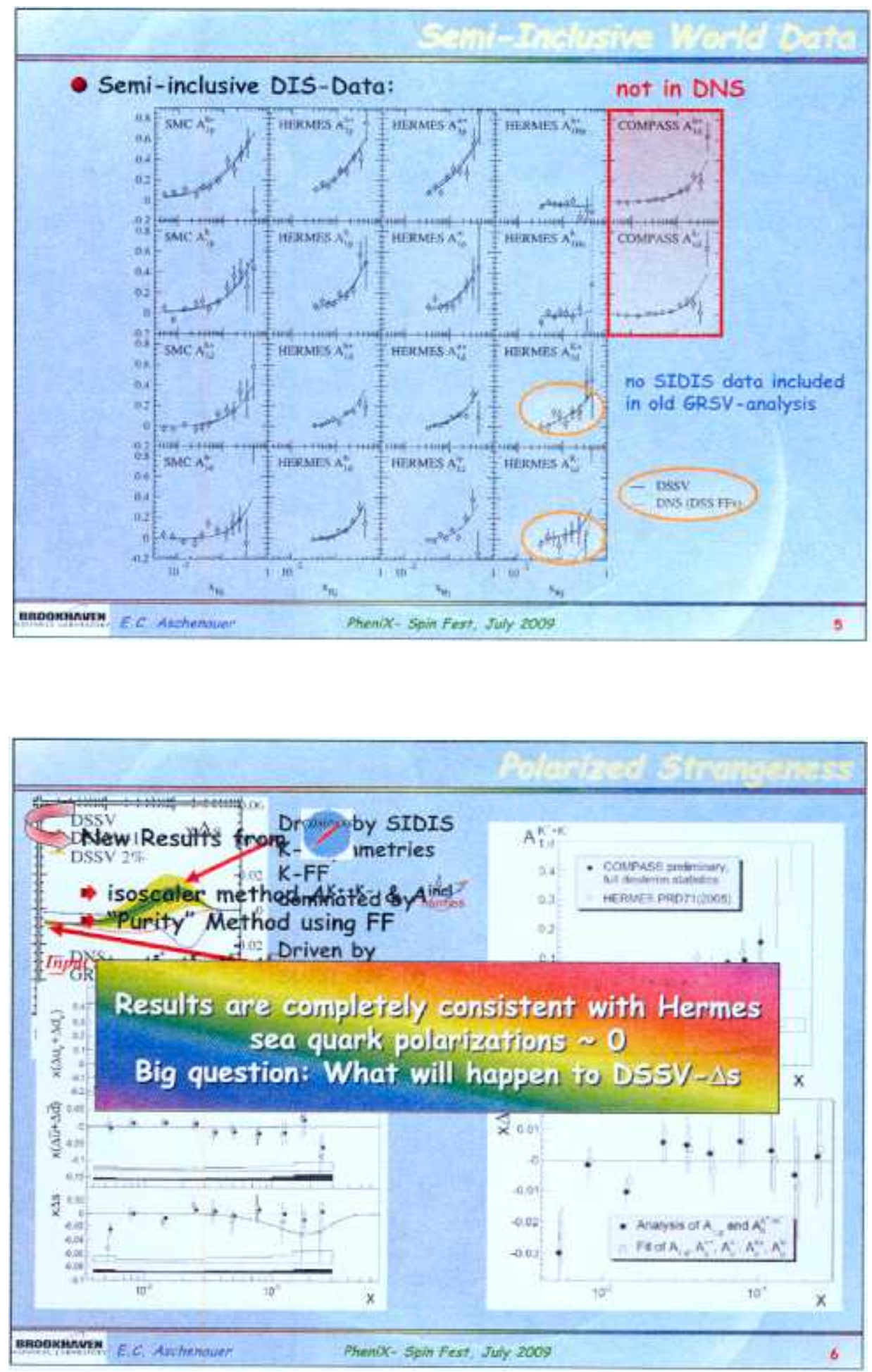

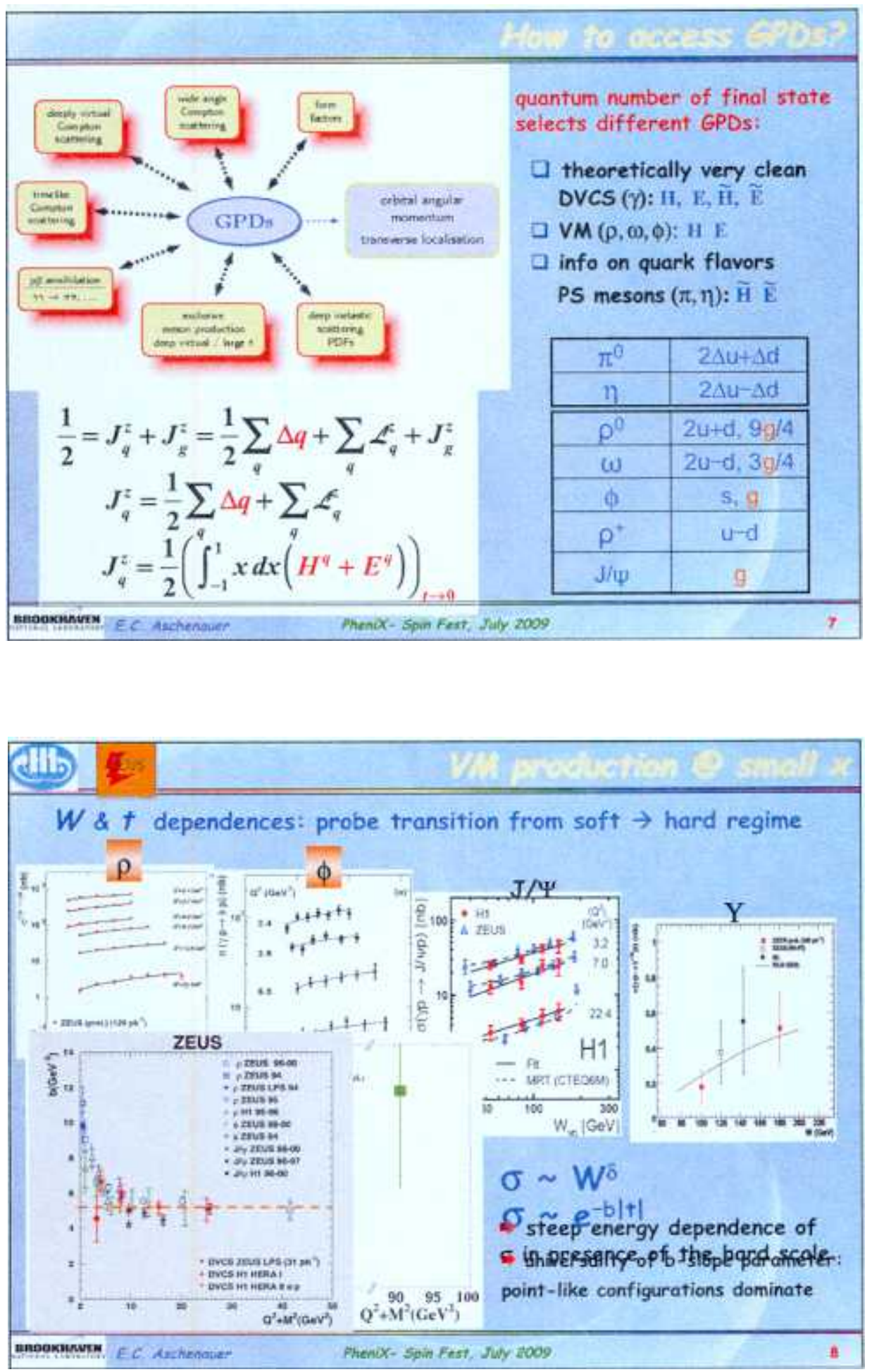

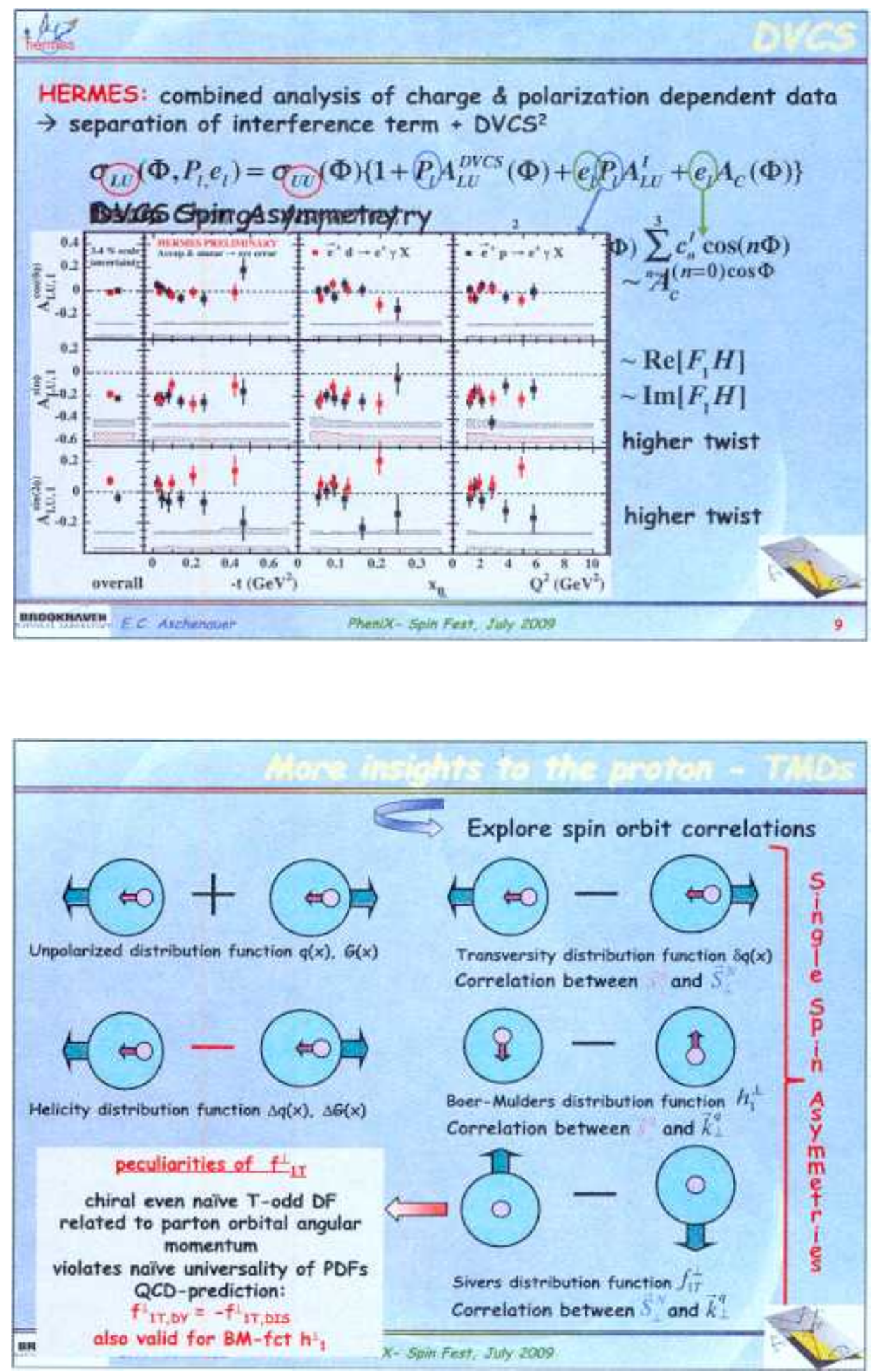

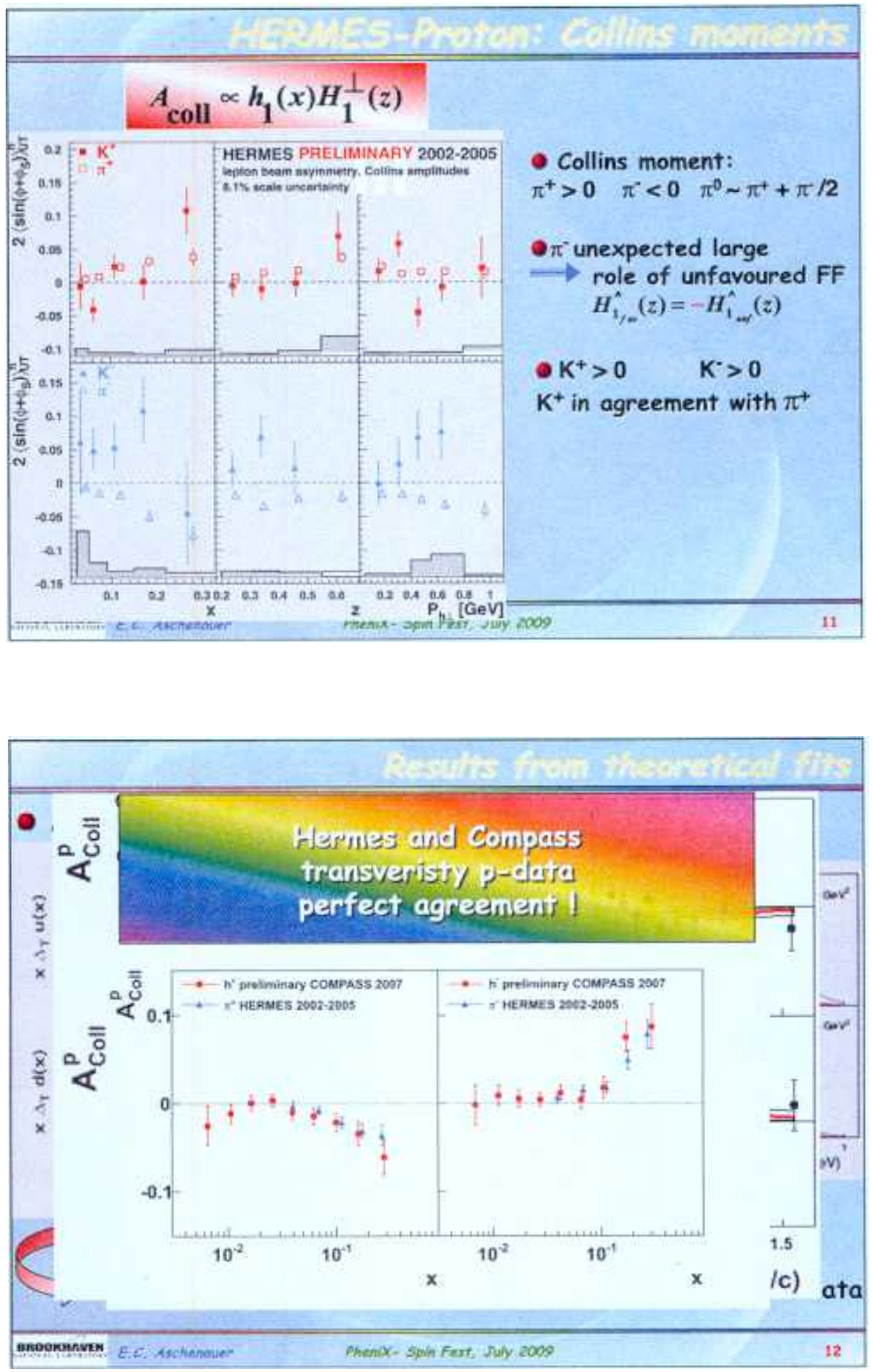

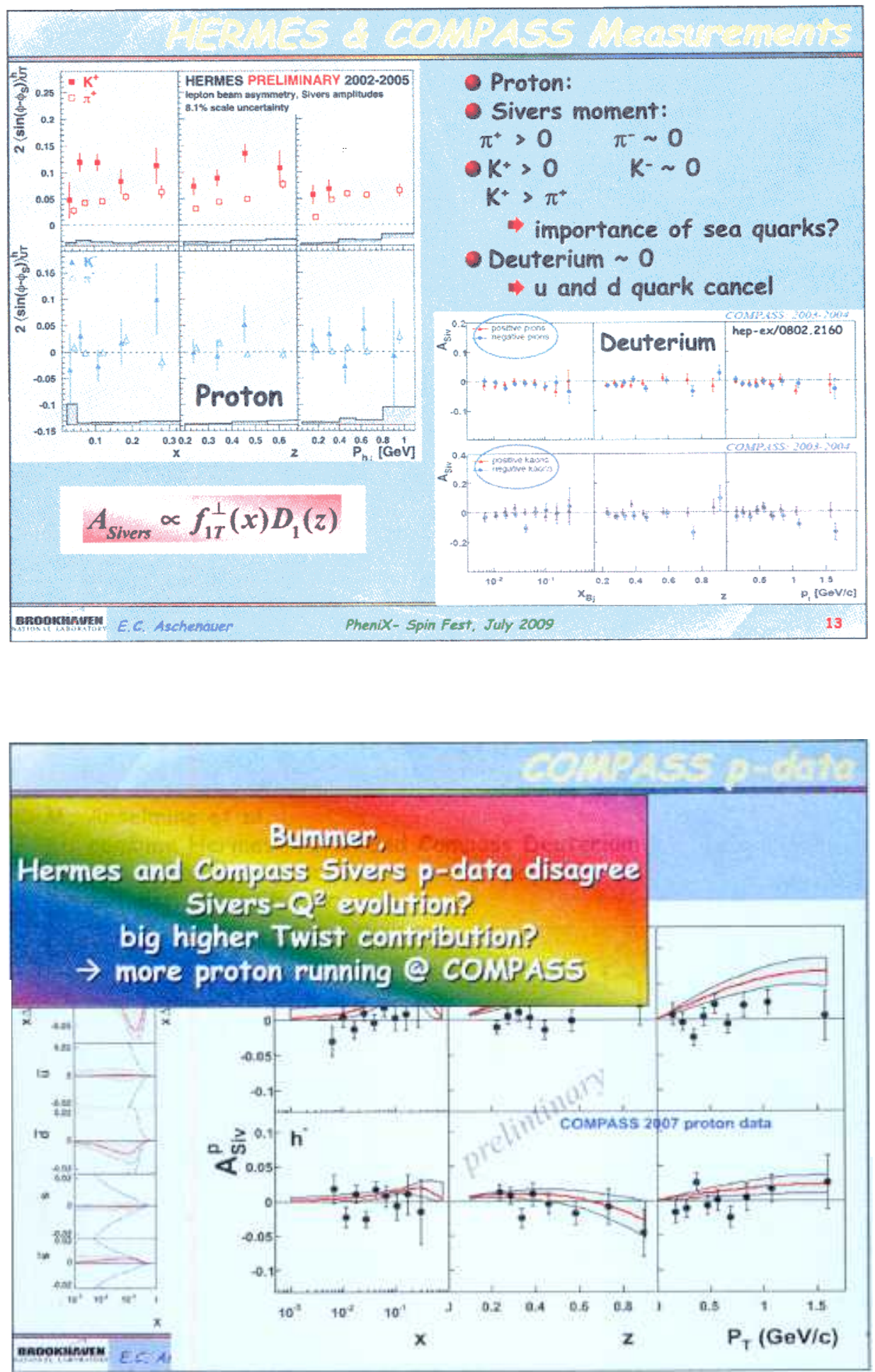

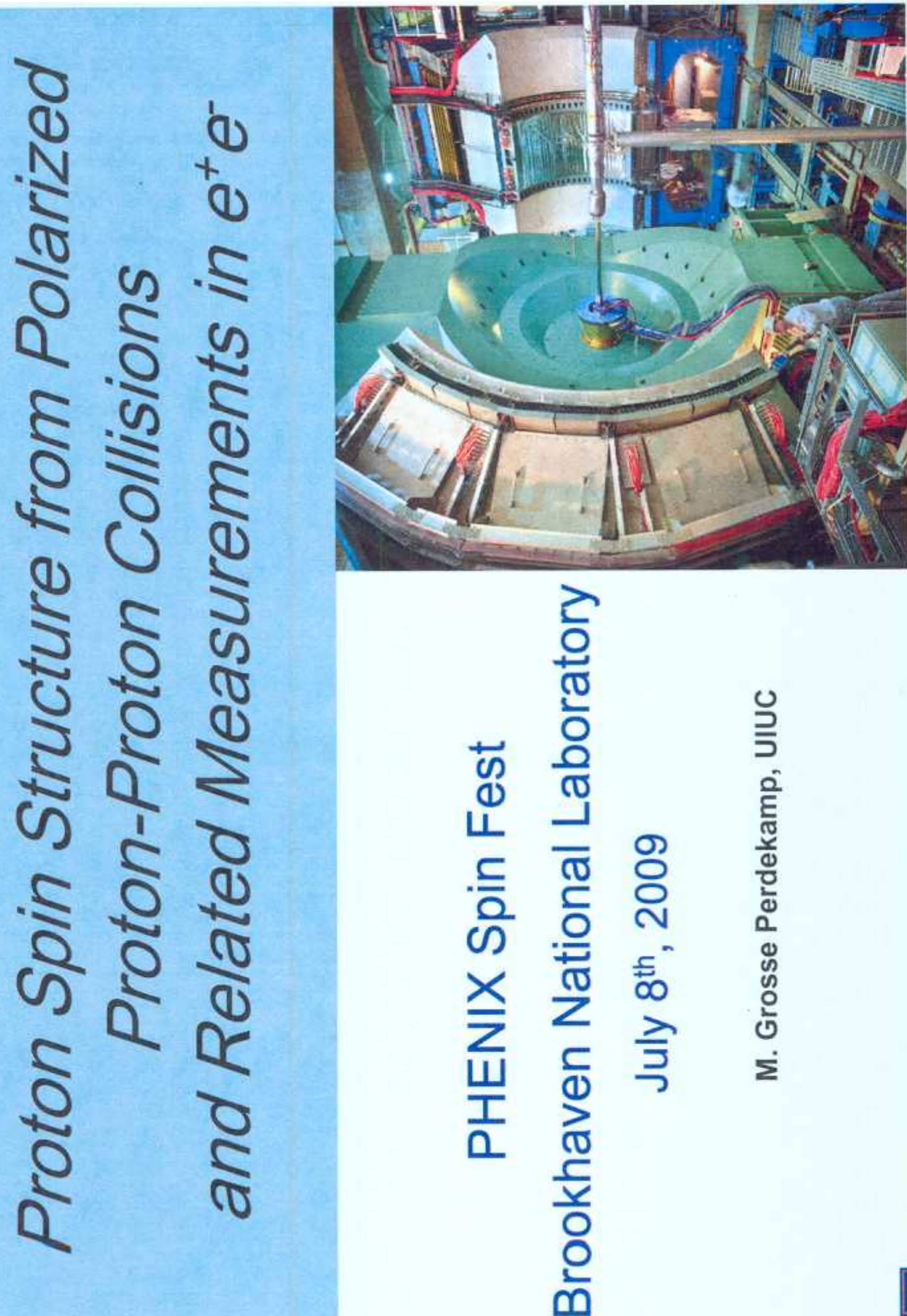


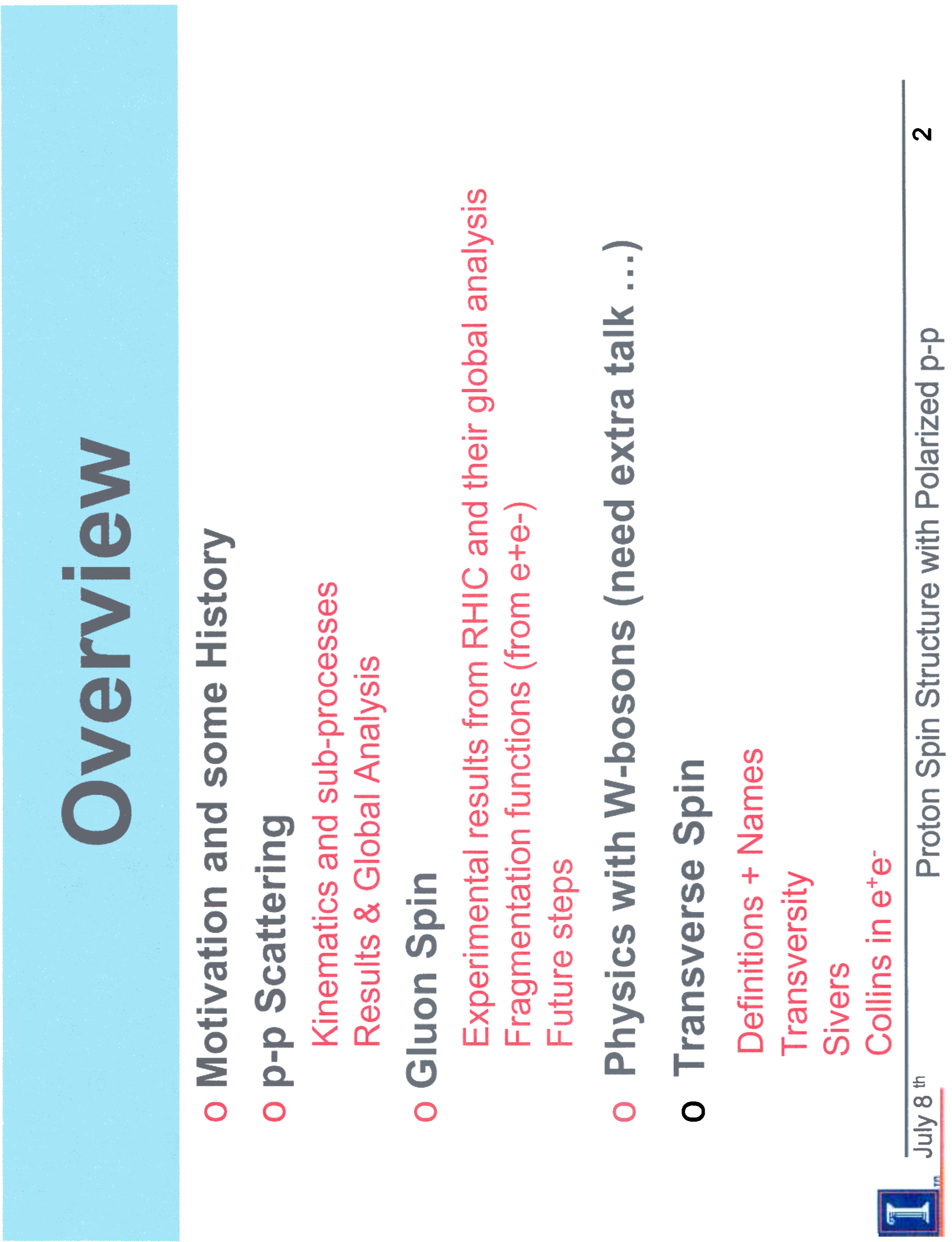




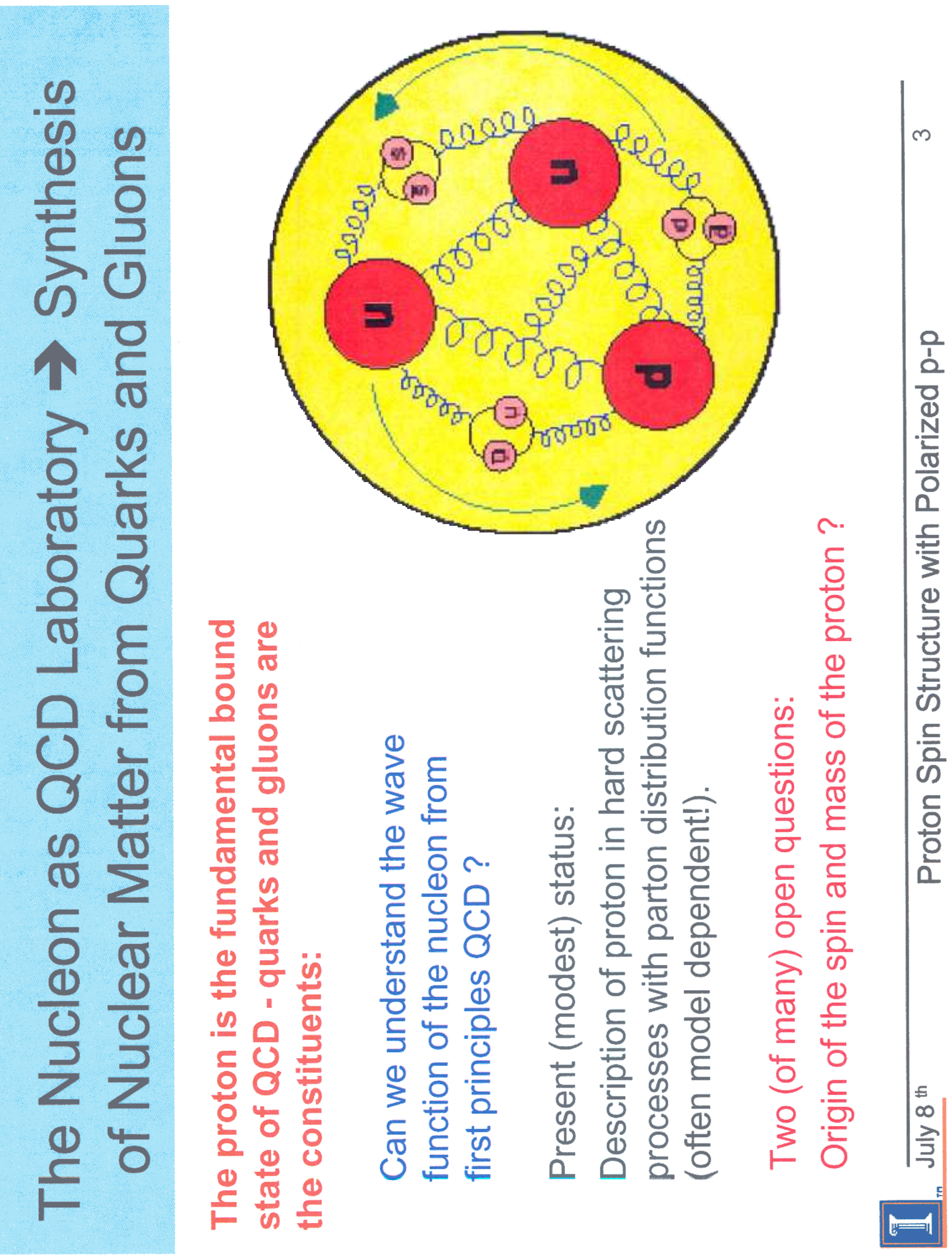




\section{The Rabi School of Physics $\Rightarrow$ Structure of Atomic Matter

Rabi maintained molecular beam laboratory at Columbia University with strong emphasize on the development of new experimental technology.

Rabi, Nobel Prize 1944

Idea $\Rightarrow$ Field new, precise instrumentation to study fundamental questions of physics.

Example: Precision Measurements of "Hydrogen Spin Structure"

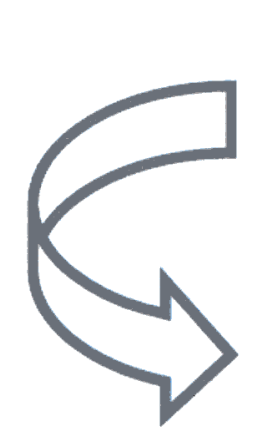
g-2 of the electron, P. Kusch Lamb shift, W. E. Lamb

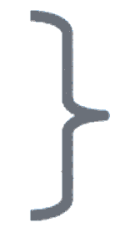

Nobel Prize 1955

Dirac Theory $\rightarrow$ QED

Tomonaga, Schwinger, Feynman

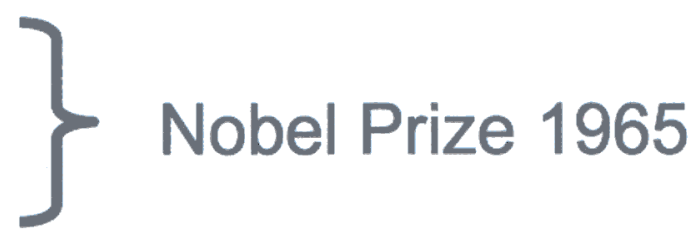




\section{Rabi School of Physics and QED}

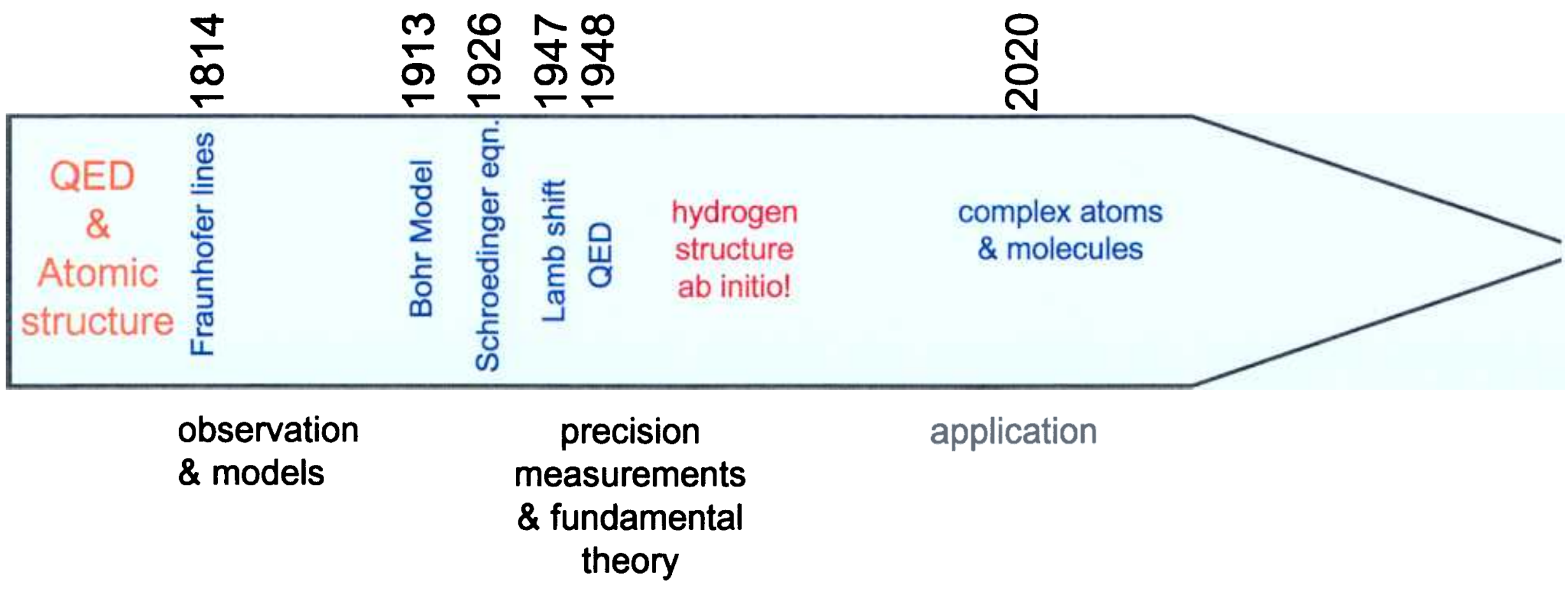




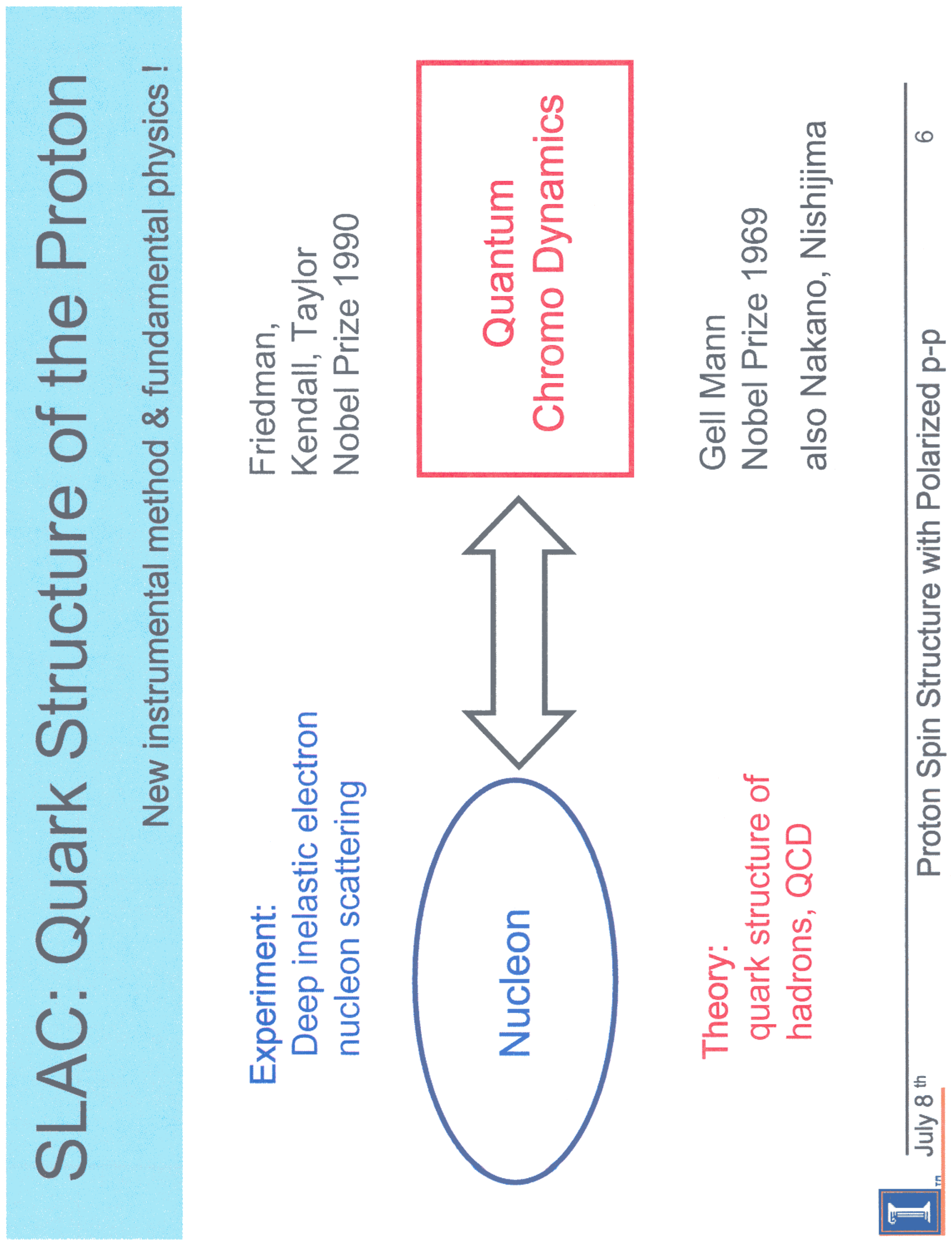




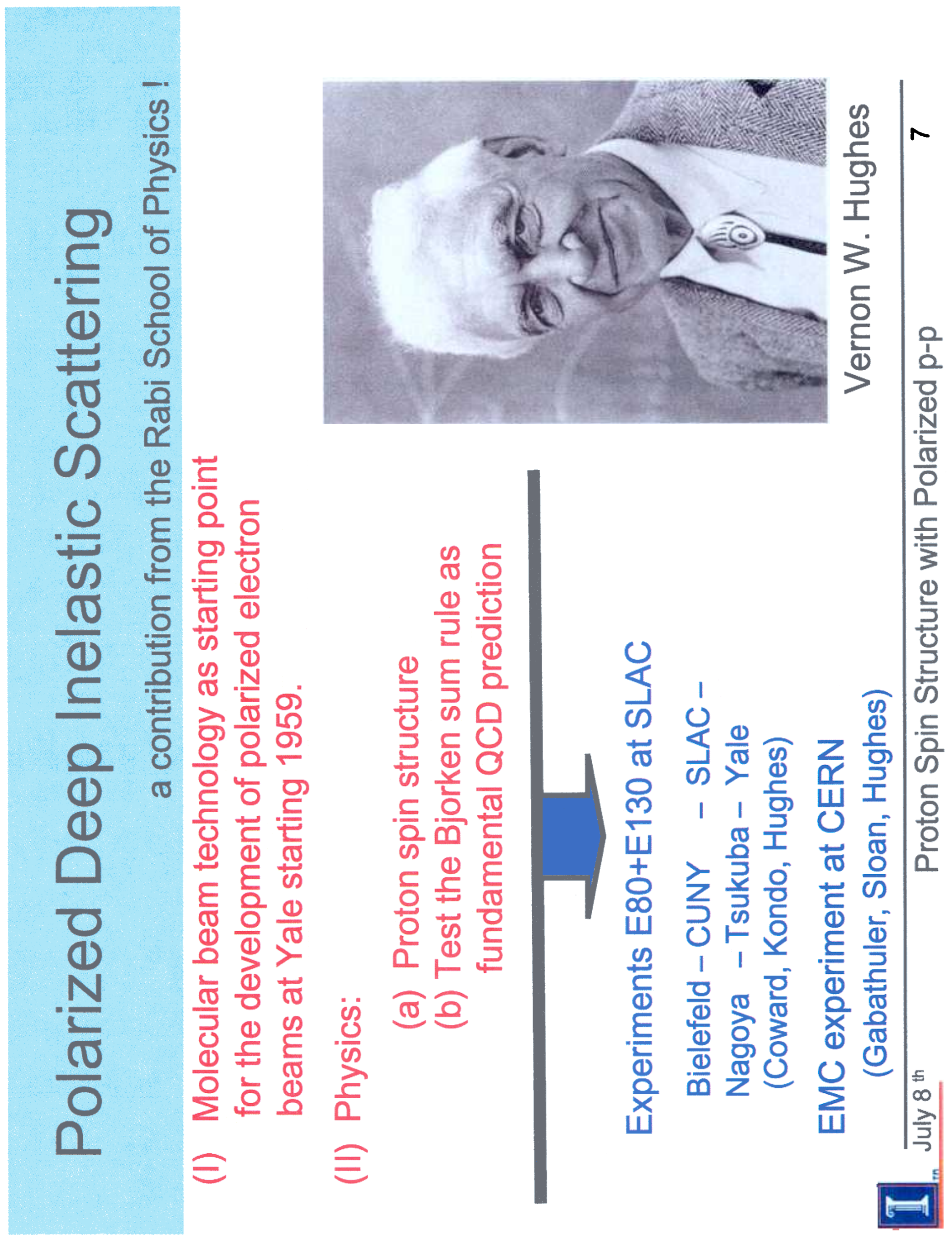




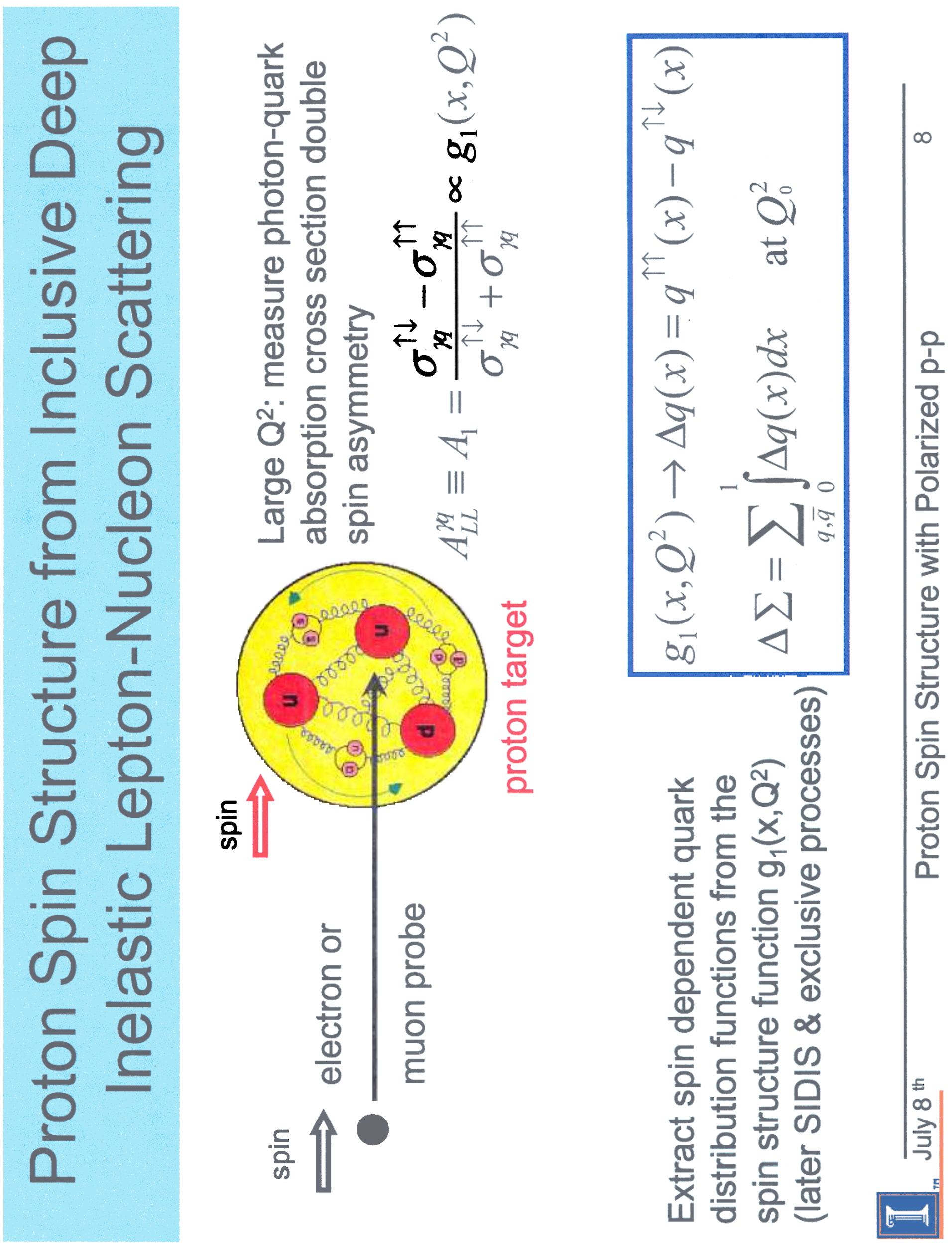




\section{Triggering in Particle Physics Experiments}

J. Lajoie, lowa State University

The role of a trigger in a particle physics experiment is to efficiently select rare physics events while suppressing background events. The degree to which this must be accomplished is determined by the requirements of the experiments data acquisition system (DAQ) and the ability to archive data to permanent storage. The trigger system must ultimately reduce in the input, or physics event rate, to the rate at data acquisition rate. These two rates can often differ by many orders of magnitude, and the challenge for the experimenter is to achieve this reduction while simultaneously maintaining a high efficiency for physics events of interest.

Experimental constraints determine the optimal approach in designing a triggers system. Cosmic rays experiments have no fixed timing structure, while accelerator experiments can take advantage of the accelerator RF structure to provide fixed time references. Fixed target experiments typically deal with an extracted beam cycle of order $\mathrm{Hz}$, with microstructure within the extracted beam that may have Mhz components. At collider experiments, bunch spacing determines the relevant timescale for a trigger decision. Electron-positron colliders typically have physics event rates of a few hundred $\mathrm{Hz}$, but are dominated by beam backgrounds. Hadron colliders have many soft QCD processes providing backgrounds to the trigger, while the desired events are relatively rare. In addition, high luminosities at hadron colliders mean that multiple interactions per bunch crossing must also be dealt with.

A given trigger system can be characterized by its efficiency for the events of interest, and it deadtime. Deadtime is defined as the fraction of the time the detector is unavailable to capture a physics event, and if determined by the trigger decision time and the readout time of the DAQ. Minimizing deadtime is a critical parameter in modern particle physics experiments, as a reduction in deadtime translates directly into more events per unit time (or dollar of running time spent). Modern trigger systems use pipelining of the trigger and front-end electronics to provide an essentially "deadtimeless" first level trigger for the experiment.

Often the required rate reduction cannot be accomplished in a single trigger step, with the limited information available at an early stage in the trigger process. This has led to the evolution of the multilevel trigger system, in which the trigger decision is refined at higher levels as the input rate to each level is reduced. This approach permits increasingly sophisticated trigger decisions at higher levels which make use of the full detector data in the trigger decision.

The development of trigger systems for particle physics experiments has been driven by increasing commercial availability of commodity hardware (FPGA's, PC's, networking, high-speed communication links, etc.) This trend will likely continue in the future, with only part of the first-level trigger system continuing to be designed in custom hardware, and full detector readout occurring at earlier stages in the trigger process. 


\section{Triggering in Particle Physics Experiments}

- Why Trigger?

- Early Experiments

- Experimental Constraints

Cross Sections and Luminosity

- Electron+positron, hadron machines

Multiple Interactions

- Signatures in Detectors

- Multilevel Trigger Systems

- Pipelined Trigger Systems

- Components of Modern Trigger Systems

- Hardware Implementation

- The PHENIX Trigger System

- Efficiency and Deadtime

- Minimizing deadtime

- Queuing Theory

- The (Near) Future

- Trigger/DAQ at LHC

Material drawn heavily from IEEE NSS

- "Non-Traditional" Expts. 2002 Workshop by Peter J. Wilson 


\section{Experimental Constraints}

Different experiments have very different trigger requirements due to different operating environments

Timing structure of beam

Rate of producing physics signals of interest

Rate of producing backgrounds

- Cosmic Ray Expts - no periodic timing structure, background/calibration source for many other experiments.

- Fixed Target Expts - close spacing between bunches in train which comes at low rep rate $(\sim \mathrm{Hz})$

- Backgrounds from undesirable spray from target

Cosmics are particularly a background for neutrino beams

- $\mathrm{e}^{+} \mathrm{e}^{-}$collider - very close bunch spacing (few nsec), beam gas and beam wall collisions

- ep collider - short bunch spacing (96ns), beam gas backgrounds

- pp/ppbar collider-modest bunch spacing (25-400ns), soft QCD 


\section{Efficiency and Deadtime I}

The goal of trigger and DAQ is to maximize the amount data sent to storage (for later analysis) for a desired process with minimal cost:

$$
\varepsilon=\varepsilon_{\text {operations }} * \varepsilon_{\text {trigger }} *(1 \text {-deadtime })
$$

-Relevant efficiency is for events that will be useful for later analysis:

$$
\varepsilon_{\text {trigger }}=N_{\text {good }}(\text { accepted }) / N_{\text {good }}(\text { produced })
$$

- For low rate process (e.g. e+e- to hadrons, Higgs production at Tevatron or LHC) try to accept all signal in trigger $\square$ Maximize efficiency

-Deadtime is due to fluctuations when the rate into a stage of the trigger (or readout) approaches the rate it can handle (busy). Simple case of no buffering:

$$
\text { deadtime }=(\text { Input Rate }) *(\text { Execution Time })
$$

- Buffering incoming data reduces dead time, more buffering less dead time

- If < Incoming Rate> > 1/<Execution Time>, dead no matter what!

-Minimizing dead-time helps all processes (and all budgets!)

- $1 \%$ of machine time $* 1$ year $=\$ \$ \$ \$$ 


\section{Multi-Level Trigger Systems}

\section{High Efficiency $\longrightarrow$ Large Rejection}

- Often can't achieve necessary rejection in a single triggering stage

- Reject in steps with successively more complete information

L0 - very fast ( $<$ bunch $x$-ing), very simple, usually scint. (TOF or Luminosity Counters)

(Few expts use a LO anymore)

L1 - fast ( few $\mu s)$ with limited information, hardware

L2 - moderately fast ( $10 \mathrm{~s}$ of $\mu \mathrm{s})$,

hardware and sometimes software

L3 - Commercial processor(s)

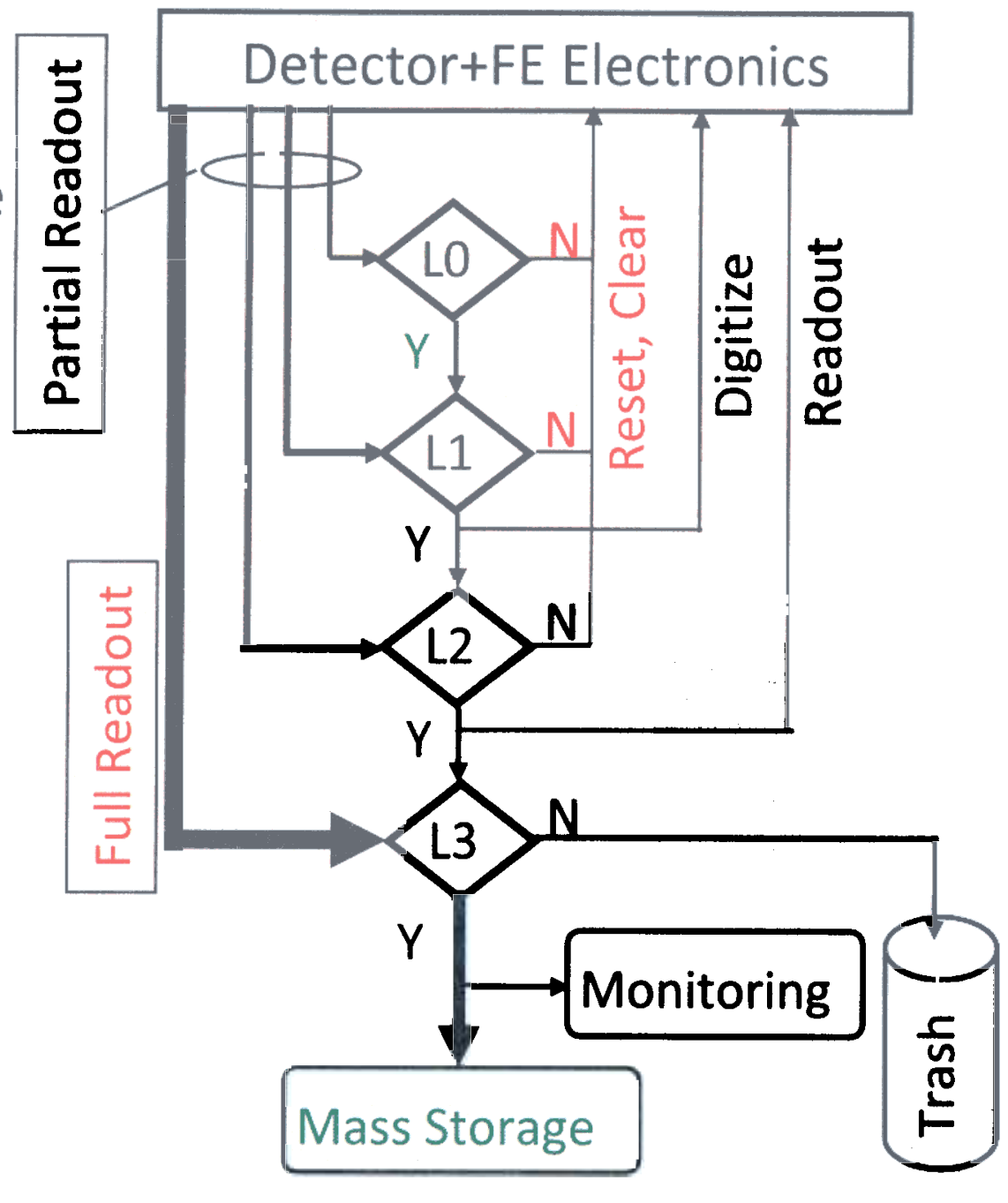




\section{Trigger and FEE Pipelining : ZEUS, H1}

Poor vacuum due to synchrotron radiation. Large proton-beam background : $\sigma_{\mathrm{pp}} \gg \sigma_{\mathrm{ep}}$ Beam-gas rate $\sim 100 \mathrm{kHz}$ (10 $\mu \mathrm{s})$

Bunch crossing rate $10.41 \mathrm{MHz}$ ( $96 \mathrm{~ns}$ ) Can't make the decision in one step! Solution is to pipeline the FEE and trigger.

Three-Level Trigger

- L1 (FLT): Hardware triggers starts readout (digitization)

- $\quad$ L2 (SLT): Software trigger with distributed processors

starts event building

- $\quad$ L3 (TLT): Software trigger in a single processor

starts data storage

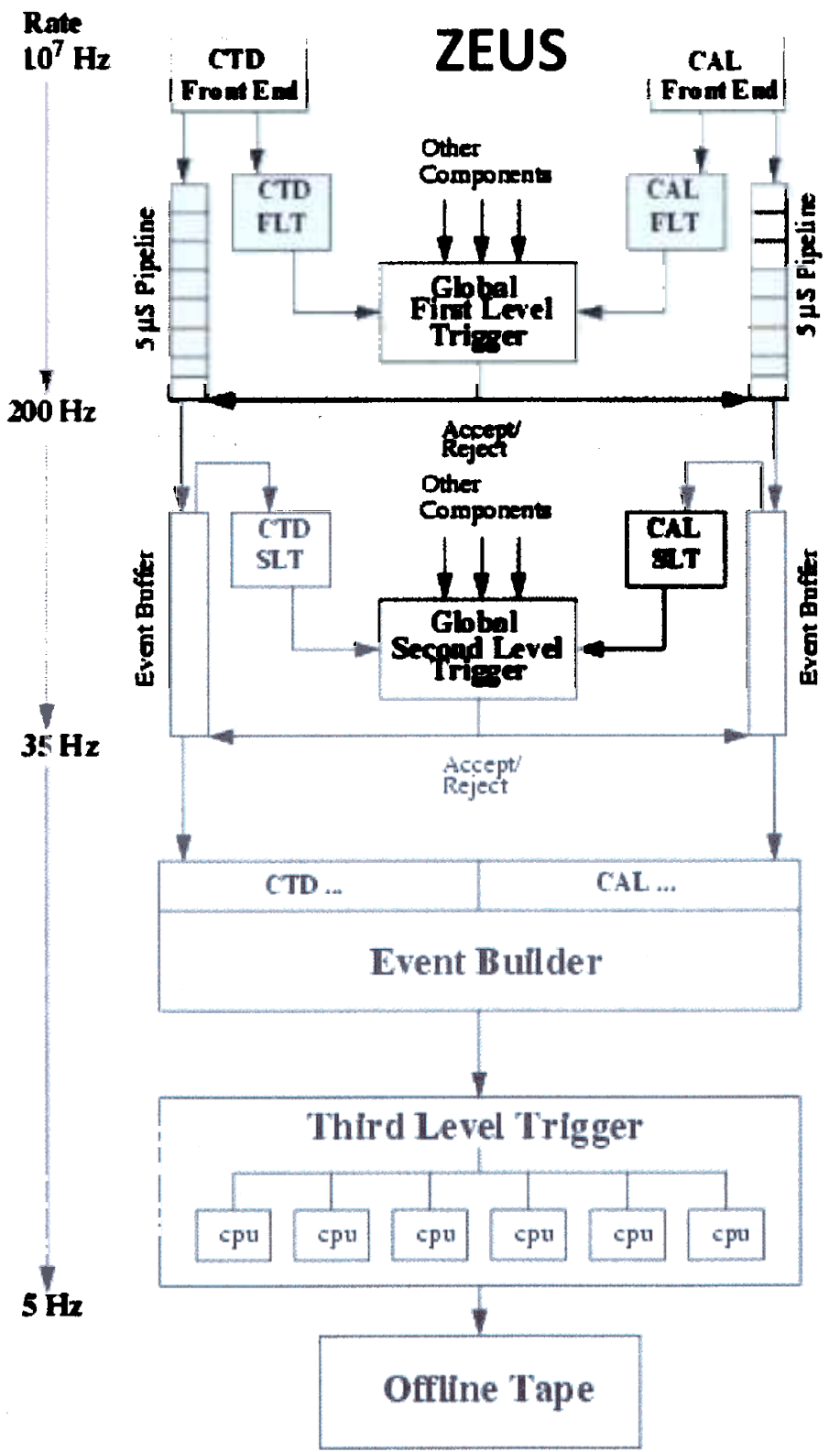


PYTHIA at RHIC - Helen Caines, Yale University, July $13^{\text {th }} 2009$

PYTHIA is based on the Lund Model of hadronization via strings, which was started by the Lund Theory Group in 1978. The Lund model assumes that all final state hadrons stem from the force between partons and not the partons themselves. Due to the self-interaction of gluons (the force carriers) the field lines between partons form narrow colored tubes, or strings. These strings that fragment causing a spray of particles between separating partons - i.e. a jet. PYTHIA models the whole of a pp event by splitting the event into several distinct parts

Hard sub-processes, which are described via matrix elements.

Resonance decays (such as the $W$ ), which are associated with the hard subprocess.

Initial State Radiation (ISR), via space like parton showers.

- Final State Radiation (FSR), via time like parton showers.

- Multiple Parton Interactions (MPI) with their ISR and FSR.

- Beam-Beam remnants.

- Reconnection of all objects via color confinement strings. Hadronization and decay of unstable particles.

The jet properties are well modeled at RHIC despite there being significant gluons scattering at these energies and gluon FF not being well measured. The intrinsic $\mathbf{k}_{\mathbf{T}}$ of the proton used in PYTHIA was extracted from CDF $1.8 \mathrm{TeV}$ data on the $\mathrm{Z}$ boson, a value of $2.1 \mathrm{GeV} / \mathrm{c}$ is used. This seems low compared to values extracted from dihadron correlations by PHENIX which is $\left\langle\mathrm{kT}^{2}>2.68 \pm 0.04 \mathrm{GeV} / \mathrm{c}\right.$.

By studying the underlying event it appears that there is very little large angle ISR/FSR at RHIC. This is in stark contrast to the significant contributions measured at the Tevatron. To describe all the details of the underlying event, including the multiplicity dependence, MPI are required. The probability of MPI is best simulated when the proton is assumed to have double-Gaussian hard core matter distribution. More "central" events have harder scattering and more MPI leading to a rising mean pr with increased multiplicity in the event. RHIC data is helping constrain the collision energy dependence of MPI. The activity in the RHIC underlying events shows that the old default scaling value in PYTHIA of $\varepsilon=0.16$ is incorrect and $\varepsilon=0.25$ should be used instead. The new value results in increased activity at RHIC but a significant decrease of $26 \%$ at the LHC.

The strange baryons and heavy flavor production are currently not well modeled by PYTHIA. A different K-factor (a simple scaling factor used to take into account the fact that PYTHIA is only a LO calculation) is required to reproduce the measured $p_{T}$ spectra resulting in the inability to simulate the light-flavor hadrons at the same time as the particles containing heavier $(s, c, b)$.

For details on the latest and greatest tune please take a look at arXiv:0905.3418 and the PYTHIA manual available from their webpage. 


\section{PYTHIA at RHIC}

\section{Helen Caines - Yale University}

PHENIX Spinfest School BNL

Outline

$12^{\text {th }}$ July 2009

- Why study $p+p$

- How PID is done

- Min-bias distributions

- Jets

- Summary

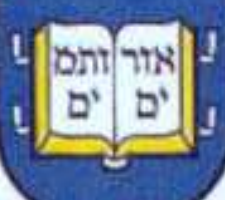

LUXETVERITIS 


\section{Intrinsic $\mathrm{k}_{\mathrm{T}}$ at $\mathrm{RHIC}$}

- STAR di-jet reconstruction

$\left\langle\mathrm{k}_{\mathrm{T}}{ }^{2}\right\rangle=2.3 \pm 0.4 \pm{ }_{1.11}^{0.67} \mathrm{GeV} / \mathrm{C}$
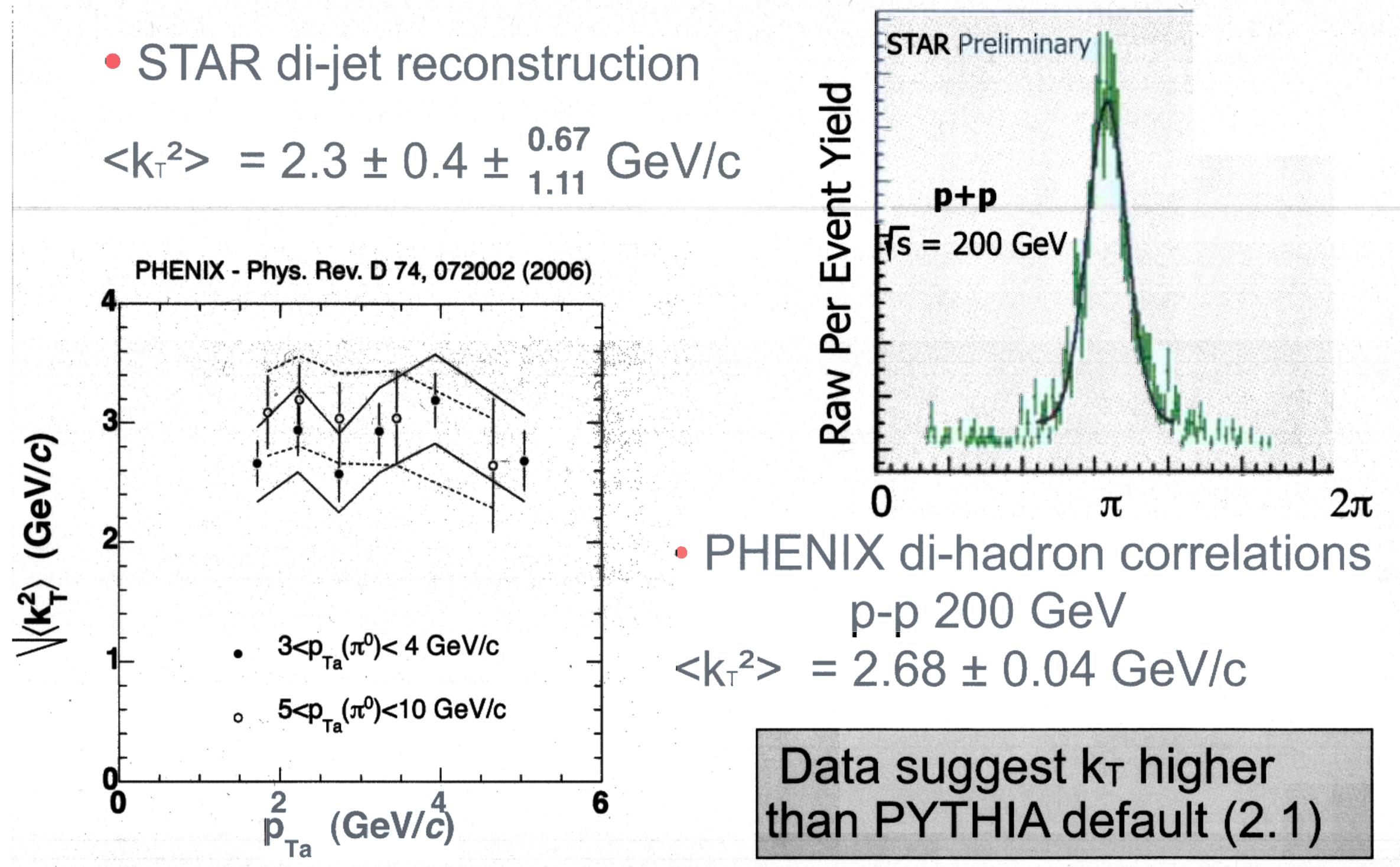

- PHENIX di-hadron correlations

p-p $200 \mathrm{GeV}$

$\left\langle\mathrm{k}_{\mathrm{T}}^{2}\right\rangle=2.68 \pm 0.04 \mathrm{GeV} / \mathrm{c}$

Data suggest $\mathrm{k}_{\mathrm{T}}$ higher than PYTHIA default (2.1) 


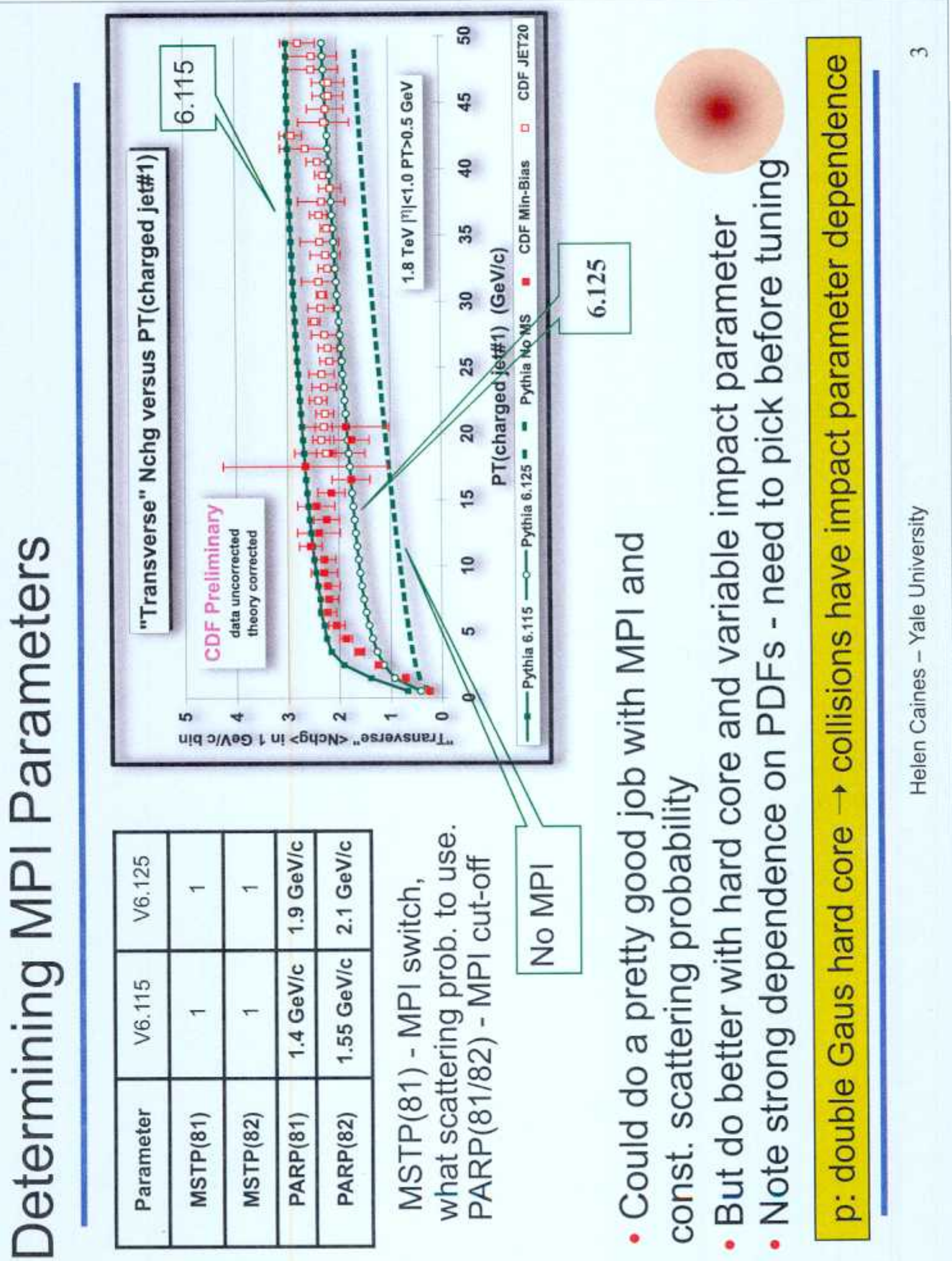




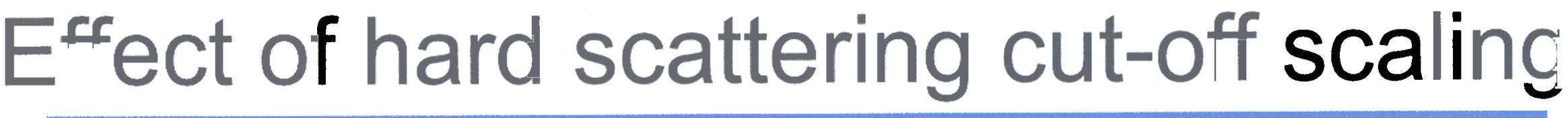

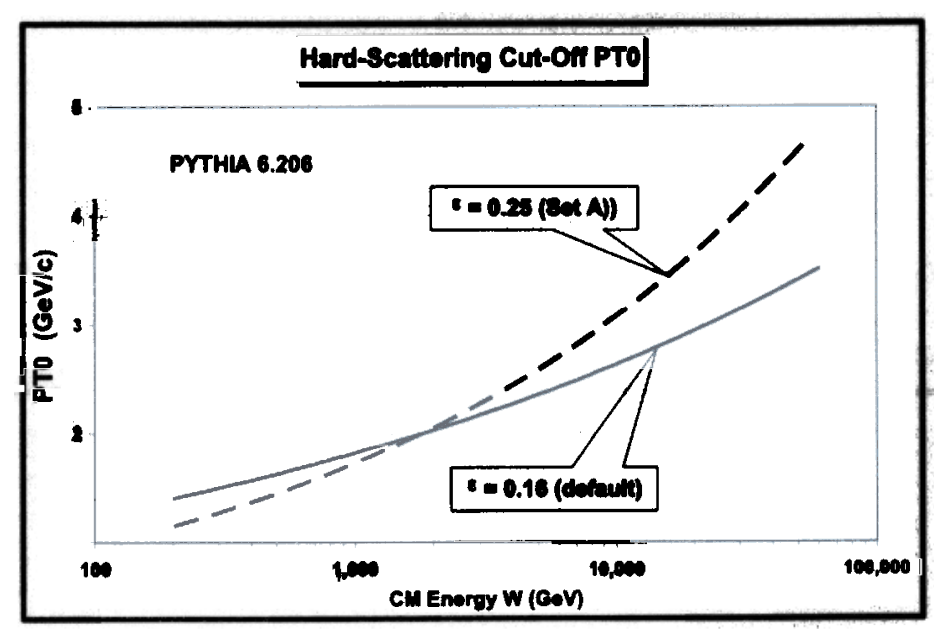

- Increasing $\varepsilon$ creates smaller energy dependence for UE - remember pivot point at $1.9 \mathrm{TeV}$

• $\varepsilon=0.16$ (DWT) $\rightarrow 0.25$ (DW) (suggested by $630 \mathrm{GeV}$ Tevatron) $\rightarrow 35 \%$ more RHIC, $26 \%$ less LHC
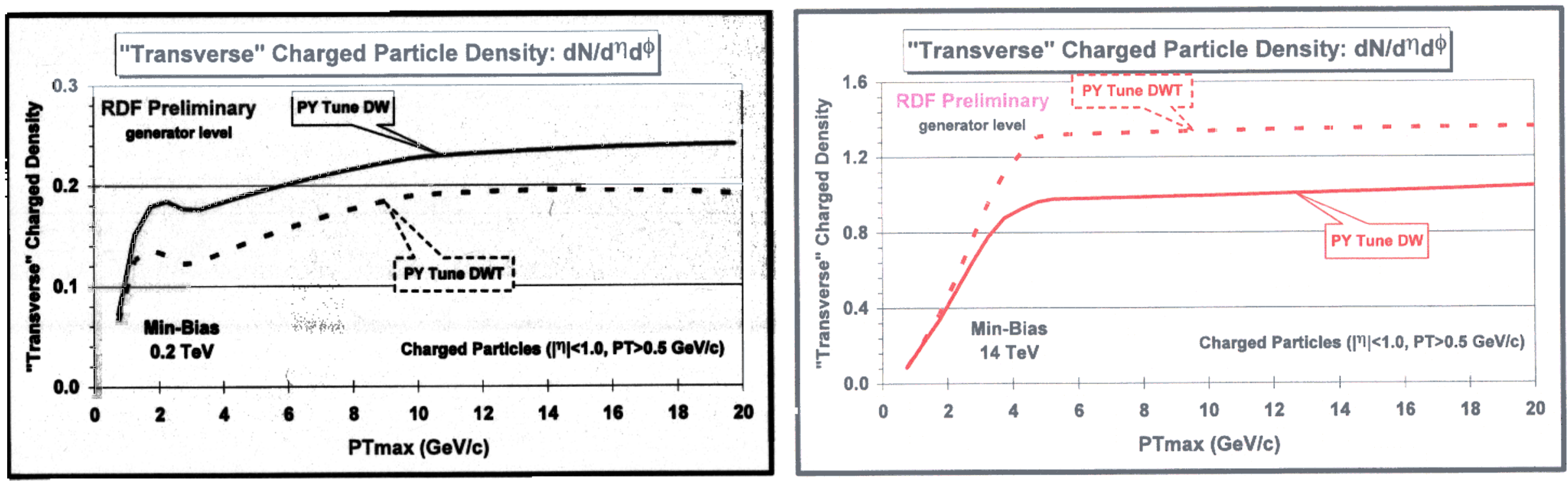


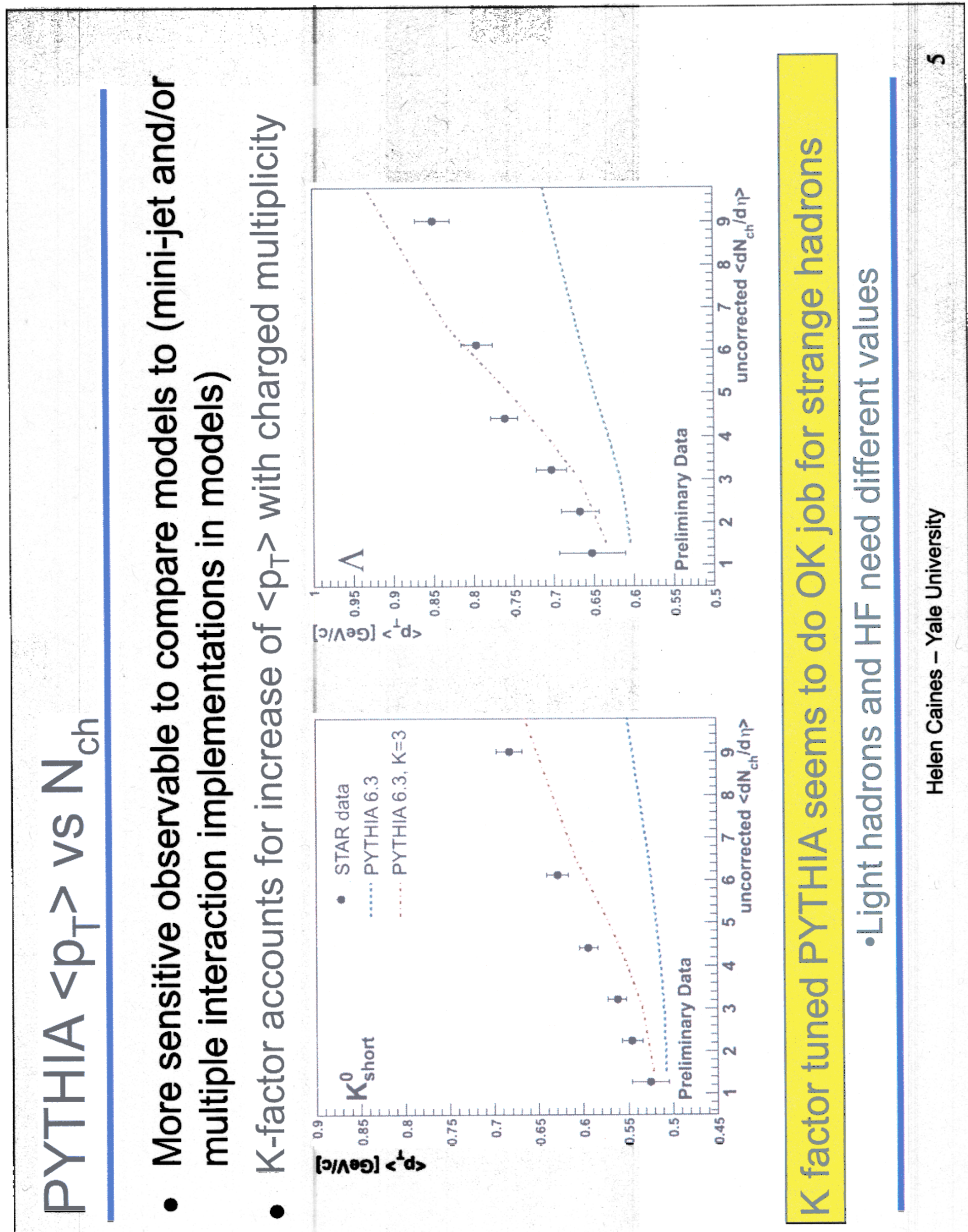




\section{Perugia Tunes}

- The major changes to PYTHIA for

Perugia tunes

- arXiv:0905.3418

\begin{tabular}{|c|c|c|c|c|c|c|c|c|c|}
\hline Parameter & Type & SOA-Pro & $\mathrm{P}-0$ & P-HARD & P-SOFT & $\mathrm{P}-3$ & P-NOCR & P-X & P-6 \\
\hline $\operatorname{MSTP}(51)$ & PDF & 7 & 7 & 7 & 7 & 7 & 7 & 20650 & 10042 \\
\hline $\operatorname{MSTP}(52)$ & PDF & 1 & 1 & 1 & 1 & 1 & 1 & 2 & 2 \\
\hline $\operatorname{MSTP}(64)$ & ISR & 2 & 3 & 3 & 2 & 3 & 3 & 3 & 3 \\
\hline PARP (64) & ISR & 1.0 & 1.0 & 0.25 & 2.0 & 1.0 & 1.0 & 2.0 & 1.0 \\
\hline $\operatorname{MSTP}(67)$ & ISR & 2 & 2 & 2 & 2 & 2 & 2 & 2 & 2 \\
\hline PARP (67) & ISR & 4.0 & 1.0 & 4.0 & 0,5 & 1.0 & 1.0 & 1.0 & 1.0 \\
\hline $\operatorname{MSTP}(70)$ & ISR & 2 & 2 & 0 & 1 & 0 & 2 & 2 & 2 \\
\hline PARP (62) & ISR & - & - & 1.25 & - & 1.25 & - & - & - \\
\hline PARP (81) & ISR & - & - & - & 1.5 & - & - & - & - \\
\hline $\operatorname{MSTP}(72)$ & ISR & 0 & 1 & 1 & 0 & 2 & 1 & 1 & 1 \\
\hline PARP (71) & FSR & 4.0 & 2.0 & 4.0 & 1.0 & 2.0 & 20 & 2.0 & 2.0 \\
\hline PARJ (81) & FSR & 0.257 & 0.257 & 0.3 & 0.2 & 0.257 & 0.257 & 0.257 & 0.257 \\
\hline PARJ (82) & FSR & 0.8 & 0.8 & 0.8 & 0.8 & 0.8 & 0.8 & 0.8 & 0.8 \\
\hline $\operatorname{MSTP}(81)$ & UE & 21 & 21 & 21 & 21 & 21 & 21 & 21 & 21 \\
\hline PARP ( 82) & UE & 1.85 & 2.0 & 2.3 & 1.9 & 2.2 & 1.95 & 2.2 & 1.95 \\
\hline $\operatorname{PARP}(89)$ & UE & 1800 & 1800 & 1800 & 1800 & 1800 & 1800 & 1800 & 1800 \\
\hline $\operatorname{PARP}(90)$ & UE & 0.25 & 0.26 & 0.30 & 0.24 & 0.32 & 0.24 & 0.23 & 0.22 \\
\hline $\operatorname{MSTP}(82)$ & UE & 5 & 5 & 5 & 5 & 5 & 5 & 5 & 5 \\
\hline PARP (83) & UEE & 1.6 & 1.7 & 1.7 & 1.5 & 1.7 & 1.8 & 1.7 & 1.7 \\
\hline $\operatorname{MSTP}(88)$ & BR & 0 & 0 & 0 & 0 & 0 & 0 & 0 & 0 \\
\hline PARP (79) & BR & 2.0 & 2.0 & 2.0 & 2.0 & 2.0 & 2.0 & 2.0 & 2.0 \\
\hline PARP ( 80$)$ & BR & 0.01 & 0.05 & 0.01 & 0.05 & 0.03 & 0.01 & 0.05 & 0.05 \\
\hline MSTP (91) & BR & 1 & 1 & 1 & 1 & 1 & 1 & 1 & 1 \\
\hline PARP (91) & BR & 2.0 & 2.0 & 1.0 & 2.0 & 1.5 & 2.0 & 2.0 & 2.0 \\
\hline PARP (93) & BR & 10.0 & 10.0 & 10.0 & 10.0 & 10.0 & 10.0 & 10.0 & 10.0 \\
\hline $\operatorname{MSTP}(95)$ & CR & 6 & 6 & 6 & 6 & 6 & 6 & 6 & 6 \\
\hline $\operatorname{PARP}(78)$ & CR & 0.2 & 0.33 & 0.37 & 0.15 & 0.35 & 0.0 & 0.33 & 0.33 \\
\hline $\operatorname{PARP}(77)$ & $\mathrm{CR}$ & 0.0 & 0.9 & 0.4 & 0.5 & 0.6 & 0.0 & 0.9 & 0.9 \\
\hline $\operatorname{MSTJ}(11)$ & HAD & 5 & 5 & 5 & 5 & 5 & 5 & 5 & 5 \\
\hline PARJ (21) & HAD & 0.313 & 0.313 & 0.34 & 0.28 & 0.313 & 0.313 & 0.313 & 0.313 \\
\hline PARJ (41) & HAD & 0.49 & 0.49 & 0.49 & 0.49 & 0.49 & 0.49 & 0.49 & 0.49 \\
\hline PARJ (42) & HAD & 1.2 & 1.2 & 1.2 & 1.2 & 1.2 & 1.2 & 1.2 & 1.2 \\
\hline PARJ (46) & HAD & 1.0 & 1.0 & 1.0 & 1.0 & 1.0 & 1.0 & 1.0 & 1.0 \\
\hline PARJ (47) & $\mathrm{HAD}$ & 1.0 & 1.0 & 1.0 & 1.0 & 1.0 & 1.0 & 1.0 & 1.0 \\
\hline
\end{tabular}




\title{
QCD and Spin Physics
}

\author{
M. Diehl
}

Deutsches Elektronen-Synchroton DESY, 22603 Hamburg, Germany

In these lectures I review some of the basic concepts of QCD that underly the interpretation of high-energy scattering experiments.

After a brief general introduction, the first lecture recalls the necessity of ultraviolet renormalization and then discusses some of its practical consequences. The connection between the renormalization scale dependence of perturbative results and the estimate of higher-order corrections is presented in some detail.

In the second lecture, I take the example of $e^{+} e^{-}$annihilation into hadrons to discuss a characteristic feature of QCD perturbation theory: the occurrence of soft and collinear divergences and their cancellation in suitable observables. I then recapitulate the optical theorem and discuss its practical value and some of its physical implications. Finally, I recall the notation of chirality and present a set of useful properties of Dirac spinors.

The third lecture reviews in broad lines the logic of collinear factorization, a cornerstone for the application of perturbative methods to lepton-hadron and hadron-hadron scattering. As a by-product, definitions and some properties of parton densities in QCD will be obtained. In the fourth lecture, I discuss the need to introduce a factorization scale and the corresponding scale dependence of parton densities. I give a brief account of the peculiarities of the evolution for polarized quark and gluon densities.

A different version of factorization is presented in the fifth lecture, where transverse-momentum dependent parton densities and fragmentation functions are introduced. I highlight the special role of Wilson lines in this context and show how they are related with time-reversal odd observables like the Sivers or Boer-Mulders asymmetries. Differences and similarities between collinear and $k_{T}$ factorization will be discussed, as well as their complementarity in the description of final states with moderately large $p_{T}$.

Throughout the lectures, emphasis is put on the physics underlying the theoretical methods on one hand, and on simple calculations illustrating their technical implementation on the other hand. 


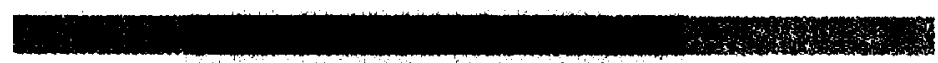

Summary of lecture 1

Renormalization

- beyond all technicalities reflects physical idea: eliminate details of physics at scales $\gg$ scale $\mu$ of problem

- dependence of observable on $\mu$ governed by RGE reflects (and estimates) particular higher-order corrections

... but not all

- prescriptions for scale choice $=$ educated guesses 


\section{Summary of lecture 2}

- perturbative calculations beyond tree level only for quantities that are IR and collinear safe $\leadsto$ dominated by large virtualities

- simplest examples: total cross sections/decay rates for colorless initial states

for jets in final state suitable (and unsuitable) observables exist

opt. theorem:

- can trade inclusive quantities $\leftrightarrow$ forward amplitudes

- generalization to non-forward amplitudes with important consequences for asymmetries

- valid for individual Feynman graphs $\rightarrow$ cutting rules 
Summary of lecture 3

Factorization

- implements ideas of parton model in QCD

- inclusion of perturbative corrections (NLO, NNLO, ...)

- field theoretical def. of parton densities and fragmentation fcts. $\leadsto$ bridge to non-perturbative QCD

- valid for specified observables in specified kinematics

- important results from general principles (power counting, ...)

- soft spectator interactions complicate analysis

for $>1$ observed hadron (SIDIS, hadron-hadron coll., ... i

- factorization proofs rather rare

- is an approximation scheme for large scales $\left(Q, p_{T}, \ldots\right)$

- certain asymmetries zero in large-scale limit, progress in calculating $\frac{1}{Q}$ suppressed ( = twist three) observables

- higher power corrections ( $\frac{1}{Q^{2}}$ etc.) in general not calculable 
6.1.

Summary of lecture 4

Evolution

- for consistency must in collinear factorization

- remove collinear kinematic region in hard scattering

- remove hard kinematic region in parton densities $\leftrightarrow$ UV renormalization

procedure introduces factorization scale $\mu$

- separates "collinear" from "hard", "object" from "probe"

- scale dependence of parton densities (and hard scattering) given by evolution equations

- for moments of parton densities get usual RGE

- special situation for first moments $\Delta \Sigma$ and $\Delta g$

- due to axial anomaly

- scheme choice exhibits limits of parton-model interpretation in full-fledged quantum field theory 


\section{Summary of lecture 5}

- transverse-momentum dependent factorization for measured small transv. mom. (or small transv. mom. differences)

- more structure in parton densities and fragm. fcts.

- $k_{T}$ dependence

- correlations between $k_{T}$ and spin of parton and/or hadron

- factorization more complicated

- soft factor

- Sudakov logarithms (but in turn no DGLAP evolution)

- nontrivial dynamics (including $T$ odd effects) from rescattering

- incorporated into Wilson lines

- best understood for $e^{+} e^{-}$, SIDIS, Drell-Yan

- $k_{T}$ factorization related with collinear factorization for high $p_{T}$ 


\section{PHENIX Spinfest 2009 at BNL List of Registered Participants}

\section{JULY $01-31,2009$}

\begin{tabular}{|c|c|c|}
\hline Name & Affiliation & E-Mail Address \\
\hline Aidala, Christine & LANL & aidala@bnl.gov \\
\hline Barish, Ken & UCR & KENNETH.BARISH@UCR.edu \\
\hline Bazilevsky, Sasha & BNL & shura@bnl.gov \\
\hline Dairaku, Seishi & KYOTO & dairaku@scphyskyoto-u.ac.jp \\
\hline Fields, Doug & UNM & fields@UNM.edu \\
\hline Goto, Yuji & RIKEN & goto@bnl.gov \\
\hline Ikeda, Yuki & RIKKYO & 08LA002X@RIKKYO.ac.jp \\
\hline Karatsu, Ken’ichi & KYOTO & karatsu@scphys.kyoto.ac.jp \\
\hline Kawall, Dave & UMASS & kawall@bnl.gov \\
\hline Kleinjan, David & UMASS & DAVID.KLEINJAN@email.UCR.edu \\
\hline Koster, John & UIUC & Jkoster4@gmail.com \\
\hline Liu, Han & LANL & nliu@RCF.RHIC.bnl.gov \\
\hline Manion, Andrew & SUNY & amanion@lc.sunysb.edu \\
\hline Mibe, Tsutomu & KEK & mibe@bnl.gov \\
\hline Nakagawa, Itaru & RIKEN & itaru@bnl.gov \\
\hline Nakano, Ken’Ichi & RIKEN & knakano@bnl.gov \\
\hline Seidl, Ralf & RBRC & siedl@bnl.gov \\
\hline Taneja, Swadhin & SUNY & taneja@skipper.physics.sunysb.edu \\
\hline Togawa, Manabu & RBRC & togawa@bnl.gov \\
\hline Wang, Xiaorong & NMSU & Xrwang@bnl.gov \\
\hline Wei, Feng & ISU & weifeng@IASTATE.edu \\
\hline Aoki, Kazuya & KYOTO & aoki@nh.scphys.kyoto-u.ac.jp \\
\hline Bathe, Stefan & RBRC & bathe@bnl.gov \\
\hline Boyle, Kieran & RBRC & boyle@bnl.gov \\
\hline Desphande, Abhay & SUNY/RBRC & abhay@bnl.gov \\
\hline Fukao, Yoshi & RIKEN & fukao@riken.jp \\
\hline Hollis, Richard & UCR & rhollis@UCR.edu \\
\hline Jiang, Xiadong & LANL & jiang@JLAB. org \\
\hline Kasai, Mikki & RIKKYO & kasai@RIKKYO.ac.jp \\
\hline Kemple, Todd & ISU & TODD.KEMPLE@ISU.edu \\
\hline Kline, Paul & SUNY & PAUL.KLEIN@stonybrook.edu \\
\hline LeeSook, Hyun & SUNY & shlee@grad.physics.sunysb.edu \\
\hline Liu, Ming & LANL & mliu@LANL.gov \\
\hline Masuike, Shunsuke & TITECH & masuke@nucl.phys.titech.ac.jp \\
\hline Miyachi, Yoshi & TITECH & miyachi@nucl.phys.titech.ac.jp \\
\hline Nakamura, Katsuro & KYOTO/RBRC & katsuro@scphys.kyoto.ac.jp \\
\hline Okada, Kensuke & RBRC & okada@bnl.gov \\
\hline Shoji, Kohei & KYOTO & shoji@nh.scphys.kyoto-u.ac.jp \\
\hline
\end{tabular}




\section{PHENIX Spinfest 2009 at BNL LIST OF REgISTERED PARTICIPANTS}

\section{JULY $01-31,2009$}

\begin{tabular}{|l|l|l|}
\hline \multicolumn{1}{|c|}{ Name } & \multicolumn{1}{c|}{ Affiliation } & \multicolumn{1}{c|}{ E-Mail Address } \\
\hline Tanida, Kiyoshi & Soul Ntl. Univ. & tanida@bnl.gov \\
\hline Vossen, Anselm & UIUC & vossen@UIUC.edu \\
\hline Watanabe, Yasushi & RIKEN & watanabe@bnl.gov \\
\hline Yang, Ruizhe & UIUC & yangrz@NPL.UIUC.edu \\
\hline & & \\
\hline & & \\
\hline & & \\
\hline & & \\
\hline
\end{tabular}


PHENIX SPINFEST SCHOOL 2009 AT BNL

Physics Bldg. 510 RBRC Conference Room 2-220, $2^{\text {nd }}$ Floor and Small Conference room $1^{\text {st }}$ floor

Agenda July $01-31,2009$

Wednesday, July $01-03$

RBRC Conference Room 2-220

Bldg. 510

Monday, July 6

RBRC Conference Room 2-220

Bldg. 510

Tuesday Morning, July 07

Small Conference Room

09:30 - 12:00

Wednesday Morning, July 08

Small Conference Room

09:30 - 12:00

Thursday Morning, July 09

Small Conference Room

$09: 30-12: 00$

Friday July 10

RBRC Conference Room

$09: 30-12: 00$

Monday Morning, July 13

Small Conference Room

$9: 30-12: 00$

Tuesday July 14 - Friday July $24^{\text {th }}$

RBRC Conference Room 2-220

Bldg. 510

Monday - Friday, July $27-31$

Small Conference Room

9:30-12:00

\section{Chair - Ralf Seidl}

Open discussion

Chair - Ralf Seidl

Open discussion

Chair - Ralf Seidl

Speaker: Elke Aschenauer, BNL

eP scattering

Chair - Ralf Seidl

Speaker: Matthias Grosse-Perdekamp, UIUC

PP scattering, (and e+e)

Chair - Ralf Seidl

Speaker: John Lajoie, ISU

Triggering in High Energy Experiments

Chair - Ralf Seidl

Open discussion

Chair - Ralf Seidl

Speaker: Helen Caines, Yale

Pythia and the Lund Fragmentation Model

Chair - Ralf Seidl

Open discussion

Chair - Ralf Seidl

Speaker: Dr. Markus Diehl, DESY

QCD Theory Lecture 


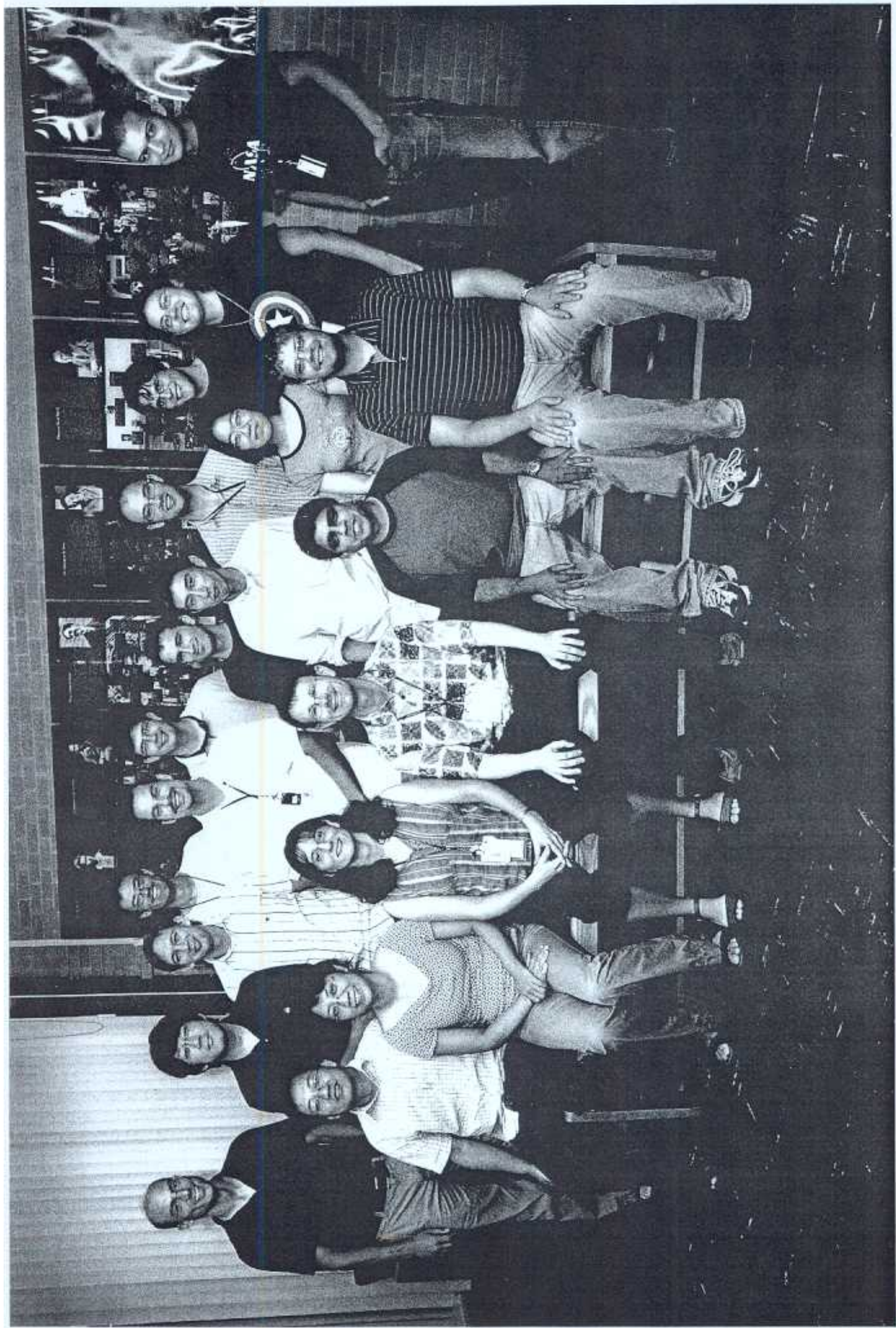


Volume 92 - PKU-RBRC Workshop of Transverse Spin Physics. June 30 - July 04, 2008. BNL-81685. 2008

Volume 91 - RBRC Scientific ReviewiCommittee Meeting. Nov. 17 \& 18, 2008. BNL-81556-2008

Volume 90 - PHENIX Splnfest School 2008 ot BNL, August 4-8, 2008. BNL-81478-2008

Vohume 89 - Understanding QGP through Spectral Functions and Eucidean Correlators, April 23-25. 2008 - BNL-81318-2008

Volume 88 - Hydrodynamics in Heary Ion Collisions and QCD Equation of Stote, April 21-22, 2008 BNL-81307-2008

Volume 87 - RBRC Scientific Revlew Committee Meeting - BNL-79570-2007

Volume 86 - Global Analysis of Polarized Parton Distributions in the RHIC Era October 8, 2007 BNL-79457-2007

Volume 85 - Patty Violoting Spin Asymmetries of RHC-BNL, April 26-27, 2007 - BNL - 79146-2007

Volume 84 - Domain Wall Fermtons of Ten Vears, March 15-17, 2007 - BNL-77857-2007

Volume 83 - QCD in Extreme Conditions, July 31-August 2, 2006 - BNL-76933-2006

Volume 82 - RHIC Physics in the Context of the Standard Model, June 18-23, 2006 - BNL-768632006

Volume 81 - Parton Orbltal Angular Momentum (Joint RBRC/University of New Mexico Workshop) Februar 24-26. 2006 - BNL-75937-2006

Volume 80 - Con We Discover the QCD Crikcal Point at RHIC?, March 9-10, 2006 - BNL 75692-2006

Volume 79 - Strangeness in Collisions, February 16-17. 2006 - BNL-79763-2008

Volume 78 - Heavy Flovor Productions and Hot/Dense Quark Matter, December 12-14, 2005 BNL-76915-2006

Volume 77 - RBRC Scientific Review Committee Meeting, October 10-12. 2005 - BNL-52649-2005

Voume 76 - Odderon Searches at RHIC. September 27-29, 2005 - BNL-75092-2005

Volume 75 - Single Spin Asymmetries. June 1-3, 2005 - BNL-74717-2005

Volume 74 - RBRC QCDPC Computer Dedication and Symposium on RBRC QCDOC, May 26. 2005 - BNL-74813-2005

Volume 73 - Jet Correlotions of RHIC, March 10-11. 2005 - BNL-73910-2005

Volume 72 - RHIC Spin Collaboration Meetings XXXX (January 14, 2005), XXX11 (February 10, 2005). XXXII (March 11. 2005) - BNL-73866-2005

Volume 71 - Clasical and Quantum.Aspects of the Color Glass Condensate - BNL-73793-2005

Votume 70 - Stonoly Coupled Plosmas: Electromognetic. Nuclear \& Atomic - BNLL-73867-2005

Volume 69 - RBRC Sclentific Review Committee Meeting - BNL-73546-2004

Volume 68 - Workshop on the Physics Programme of the RBRC and UKQCD QCDOC Machines BNL-73604-2004

Volume 67 - High Performance Computing with BlueGene/L and QCDOC Architectures - BNLVolume 66 - RHIC Spin Collaboration Meeting XXIX October 8-9. 2004, Torino, Itaty - BNL-735342004

Volume 65 - RHIC Spin Collaborotion Meetings XXVI (July 22, 2004), XXVII (September 2, 2004) BNL-73506-2004

Volume 64 - Theory Summer Program on RHIC Physics - BNL-73263-2004

Volume 63 - RHIC Spln Collaboration Meetings XXIV (05/21/04), XXV (05/27/04), XXV (06/01/04) BNL-72397-2004

Volume 62 - New Discoverles of RHIC. MaY 14-15, 2004 - BNL-72391-2004

Volume 61 - RIKEN-TODA Mini Workshop on "Topics in Hodion Physics of RHIC", March 23-24, 2004 -

BNL-72336-2004

Vohume 60 - Lattice QCD at Fintte Temperature and Density - BNL-72083-2004

Volume 59 - RHIC Spin Colkaboration Meeting XXI, XXII, XXIH - BNL-72382-2004

Volume 58 - RHHC Spin Collaboration Meeting XX - BNL-71900-2004

Vohume 57 - Hilgh pt Pinysics ot RHIC. December 2-6, 2003 - BNL-72069-2004

Volume 50 - RBRC Scientific Review Committee Meeting - BNL-71899-2003

Volume 55 - Collective Flow and QGP Properties - BNL-71898-2003

Vohume 54 - RHIC Spin Collaborotion Meetings XVI, XVII, XIX - BNL-71751-2003

Volume 53 - Theory Shudles for Polarized pp Scottering - BNL-71747-2003

Volume 52 - RIKEN School on QCD. "Toplcs on the Proton" - BNL-71694-2003

Volume 51 - RHIC Spin Collaboration Meetings XV. XV - BNL-71539-2003

Volume 50 - High Performance Computing with $Q$ CDOC and BlueGene - BNL-71 147-2003

Volume 49 - RBRC Sclentific Review Committee Meeting - BNL-52679

Volume 48 - RHIIC Spin Collaboration Meeting XIV - BNL-71300-2003

Volume 47 - RHIC Spln Collaboration Meetings XII, XIII - BNL-71118-2003

Volume 46 - Large-Scale Computations in Nuclear Physics using the QCDOC - BNL-52678

Volume 45 - Summer Program: Current and Future Directions of RHIC - BNL-71035 
Volume 44 - RHIC Spin Collaboration Meetings VIII, IX,X,XI-BNL-711117-2003

Volume 43 - RIKEN Wnter School - Quark-Gluon Structure of the Nucleon and $Q C D$ - BNL-52672

Volume 42 - Baryon Dynamics ot RHIC - BNL-52669

Volume 41 - Hadron Structure from Lattice QCD - BNL-52674

Volume 40 - Theory Studies for RHIC-Spin - BNL-52662

Volume 39 - RHIC Spin Collaboration Meeting VI - BNL-52659

Volume 38 - RBRC Scientific Revlew Committee Meeting - BNL-52649

Volume 37 - RHIC Spin Collaboration Meeting VI (Part 2) - BNL-52660

Volume 36 - RHIC Spln Collaboration Meeting VI - BNL-52642

Volume 35 - RIKEN Winter School - Quarks, Hadrons and Nuclei - QCD Hard Processes and the Nucleon Spin - BNL-52643

Volume 34 - High Energy QCD: Beyond the Pomeron - BNL-52641

Volume 33 - Spin Physics at RHIC in Year-1 and Beyond - BNL-52635

Volume 32 - RHIC Spin Physics V - BNL-52628

Volume 31 - RHIC Spin Physics III \& IV Polarized Partons ot High Q^2 Region - BNL 52617

Volume 30 - RBRC Sclentific Review Committee Meeting - BNL-52603

Volume 29 - Future Transversity Measurements - BNL-52612

Volume 28 - Equllibrium \& Non-Equllibrium Aspects of Hot. Dense QCD - BNL-52613

Volume 27 - Predictions and Uncertainties for RHIC Spin Physics \& Event Generator for RHIC Spin

Physics III - Towards Precision Spin Physics at RHIC - BNL-52596

Volume 26 - Clrcum-Pan-Pocific RIIEN Symposium on High Energy Spin Physics - BNL-52588

Volume 25 - RHIC Spin - BNL-52581

Volume 24 - Physics Soclety of Japan Biannual Meeting Symposium on 2 CD Physics of RIKEN BNL

Research Center - BNL-52578

Volume 23 - Coulomb and Plon-Asymmetry Polarimetry and Hadronic Spin Dependence at RHIC Energles - BNL-52589

Volume 22 - OSCAR II: Predictions for RHIC - BNL-52591

Volume 21 - RBRC Scientific Review Committee Meeting - BNL-52568

Volume 20 - Gauge-Invariant Variables in Gauge Theories - BNL-52590

Volume 19 - Numerical Agorithms at Non-Zero Chemical Potential - BNL-52573

Votume 18 - Event Generator for RHIC Spin Physics - BNL-52571

Volume 17 - Hard Parton Physics in High-Energy Nuclear Collisions - BNL-52574

Volume 16 - RIKEN Winter School - Structure of Hadrons - Introduction to QCD Hard Processes BNL-52569

Volume 15 - ®CD Phase Transitions - BNL-52561

Volume 14 - Quantum Fields in and Out of Equilibrium - BNL-52560

Volume 13 - Physics of the 1 Teraflop RIKEN-BNL-Columbla QCD Project First Anniversany

Celebration - BNL-66299

Volume 12 - Quarkonlum Production in Relativistic Nuclear Collisions - BNL-52559

Volume 11 - Event Generator for RHCC Spin Physics - BNL-66 116

Volume 10 - Phystes of Polarimetry at RHIC - BNL-65926

Volume 9 - High Density Matter in AGS, SPS and RHIC Collisions - BNL-65762

Volume 8 - Fermion Frontiers in Vector Lattice Gauge Theories - BNL-65634

Volume 7 - RHIC Spin Physics - BNL- 45615

Volume 6 - Quarks and Gluons in the Nucleon - BNL-65234

Volume 5 - Cotor Superconductivity, Instantons and Partty (Non?)-Conservation at High Baryon

Density - BNL-65105

Volume 4 - Inauguration Ceremony, September 22 and Non -Equillbrium Many Body Dynamics BNL-64912

Volume 3 - Hadron Spin-Flip at RHIC Energies - BNL-64724

Volume 2 - Perturbative QCD as a Probe of Hadron Structurt - BNL-64723

Volume 1 - Open Standards for Cascade Models for RHIC - E NL-64722 


\section{For information please contact:}

Ms. Susan P. Foster

RIKEN BNL Research Center

Experimental Group Administrator

Building 510A; Room 2-70

Brookhaven National Laboratory

Upton, NY 11973-5000 USA

Phone: (631) 344-5864

Fax: (631) 344-2562

E-Mail: sfoster@bnl.gov

Information on RBRC (RIKEN BNL Research Center):

RIKEN Home page: http://www.bnl.gov/riken/

RHIC Spin Group: http://www.phy.bnl.gov/rhicspin/

Spin Collaboration: http://spin.riken.bnl.gov/ 


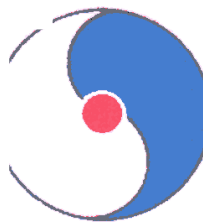

\section{RIKEN BNL RESEARCH CENTER}

\section{PHENIX SpinFest School 2009 BNL}
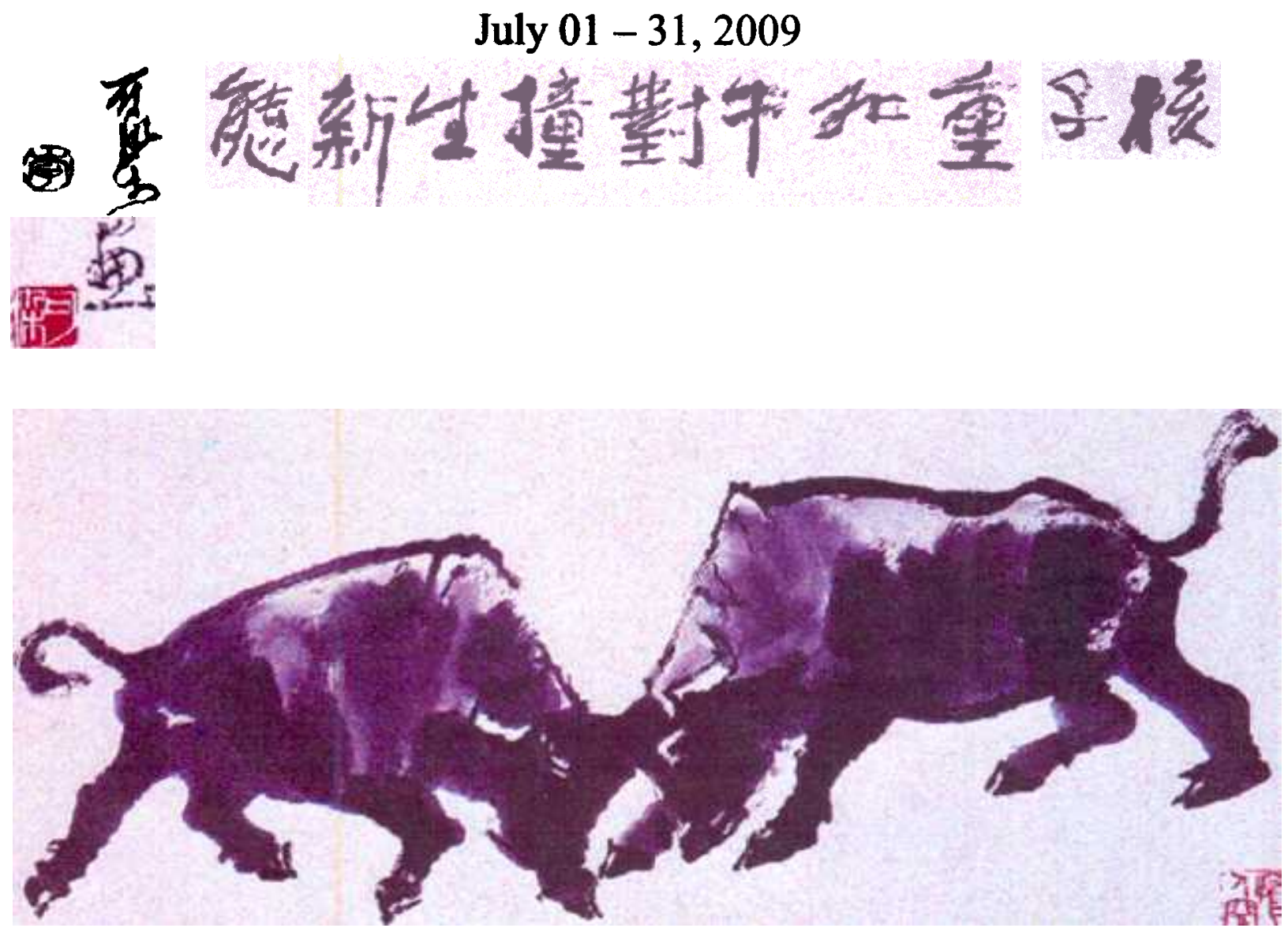

Keran

Nuclei as heavy as bulls

CopyrightoCCASTA

Through collision

Generate new states of matter.

T.D. Lee

Speakers:

Elke Aschenauer (BNI

Helen Caines (Yale)

Matthias Grosse-Perdekamp (UIUC)

John Lajoie (ISU

Markus Diehl (DESY)

Organizers:

Ralf Seidl (BNL/RBRC); Yuji Goto (RIKEN/RBRC); Kensuke Okada (BNL/RBRC) 